\title{
Multistep Hybrid Extragradient Method for Triple Hierarchical Variational Inequalities
}

\author{
Zhao-Rong Kong, ${ }^{1}$ Lu-Chuan Ceng, ${ }^{2}$ Qamrul Hasan Ansari, ${ }^{3}$ and Chin-Tzong Pang ${ }^{4}$ \\ ${ }^{1}$ Department of Mathematics, Shanghai Normal University, Shanghai 200234, China \\ ${ }^{2}$ Department of Mathematics, Shanghai Normal University, and Scientific Computing Key Laboratory of Shanghai Universities, \\ Shanghai 200234, China \\ ${ }^{3}$ Department of Mathematics, Aligarh Muslim University, Aligarh 202 00, India \\ ${ }^{4}$ Department of Information Management, Yuan Ze University, Chung Li 32003, Taiwan
}

Correspondence should be addressed to Chin-Tzong Pang; imctpang@saturn.yzu.edu.tw

Received 26 December 2012; Accepted 25 January 2013

Academic Editor: Jen-Chih Yao

Copyright (C) 2013 Zhao-Rong Kong et al. This is an open access article distributed under the Creative Commons Attribution License, which permits unrestricted use, distribution, and reproduction in any medium, provided the original work is properly cited.

\begin{abstract}
We consider a triple hierarchical variational inequality problem (THVIP), that is, a variational inequality problem defined over the set of solutions of another variational inequality problem which is defined over the intersection of the fixed point set of a strict pseudocontractive mapping and the solution set of the classical variational inequality problem. Moreover, we propose a multistep hybrid extragradient method to compute the approximate solutions of the THVIP and present the convergence analysis of the sequence generated by the proposed method. We also derive a solution method for solving a system of hierarchical variational inequalities (SHVI), that is, a system of variational inequalities defined over the intersection of the fixed point set of a strict pseudocontractive mapping and the solution set of the classical variational inequality problem. Under very mild conditions, it is proven that the sequence generated by the proposed method converges strongly to a unique solution of the SHVI.
\end{abstract}

\section{Introduction and Formulations}

Throughout the paper, we will adopt the following terminology and notations. $\mathscr{H}$ is a real Hilbert space, whose inner product and norm are denoted by $\langle\cdot, \cdot\rangle$ and $\|\cdot\|$, respectively. The strong (resp., weak) convergence of the sequence $\left\{x_{n}\right\}$ to $x$ will be denoted by $x_{n} \rightarrow x$ (resp., $x_{n} \rightarrow x$ ). We shall use $\omega_{w}\left(x_{n}\right)$ to denote the weak $\omega$-limit set of the sequence $\left\{x_{n}\right\}$; namely,

$$
\begin{aligned}
& \omega_{w}\left(x_{n}\right) \\
& \quad:=\left\{x: x_{n_{k}} \rightarrow x \text { for some subsequence }\left\{x_{n_{k}}\right\} \text { of }\left\{x_{n}\right\}\right\} .
\end{aligned}
$$

Throughout the paper, unless otherwise specified, we assume that $C$ is a nonempty, closed, and convex subset of a Hilbert space $\mathscr{H}$ and $A: C \rightarrow \mathscr{H}$ is a nonlinear mapping.
The variational inequality problem (VIP) on $C$ is defined as follows:

$$
\text { find } x^{*} \in C \text { such that }\left\langle A x^{*}, x-x^{*}\right\rangle \geq 0, \quad \forall x \in C .
$$

We denote by $\Gamma$ the set of solutions of VIP. In particular, if $C$ is the set of fixed points of a nonexpansive mapping $T$, denoted by $\operatorname{Fix}(T)$, then (VIP) is called a hierarchical variational inequality problem (HVIP), also known as a hierarchical fixed point problem (HFPP). If we replace the mapping $A$ by $I-S$, where $I$ is the identity mapping and $S$ is a nonexpansive mapping (not necessarily with fixed points), then the VIP becomes as follows:

$$
\begin{aligned}
& \text { find } x^{*} \in \operatorname{Fix}(T) \text { such that }\left\langle(I-S) x^{*}, x-x^{*}\right\rangle \geq 0 \text {, } \\
& \forall x \in \operatorname{Fix}(T) \text {. }
\end{aligned}
$$

This problem, first introduced and studied in $[1,2]$, is called a hierarchical variational inequality problem, also known as 
a hierarchical fixed point problem. Observe that if $S$ has fixed points, then they are solutions of VIP (3). It is worth mentioning that many practical problems can be written in the form of a hierarchical variational inequality problem; see, for example, [1-18] and the references therein.

If $S$ is a $\rho$-contraction with coefficient $\rho \in[0,1)$ (i.e., $\| S x-$ $S y\|\leq \rho\| x-y \|$ for some $\rho \in[0,1))$, then the set of solutions of VIP (3) is a singleton, and it is well known as a viscosity problem, which was first introduced by Moudafi [19] and then developed by $\mathrm{Xu}$ [20]. It is not hard to verify that solving VIP (3) is equivalent to finding a fixed point of the nonexpansive mapping $P_{\mathrm{Fix}(T)} S$, where $P_{\mathrm{Fix}(T)}$ is the metric projection on the closed and convex set $\operatorname{Fix}(T)$.

Let $F: C \rightarrow \mathscr{H}$ be $\kappa$-Lipschitzian and $\eta$-strongly monotone, where $\kappa>0, \eta>0$ are constants, that is, for all $x, y \in C$

$$
\|F x-F y\| \leq \kappa\|x-y\|, \quad\langle F x-F y, x-y\rangle \geq \eta\|x-y\|^{2} .
$$

A mapping $T: C \rightarrow C$ is called $\zeta$-strictly pseudocontractive if there exists a constant $\zeta \in[0,1)$ such that

$$
\begin{array}{r}
\|T x-T y\|^{2} \leq\|x-y\|^{2}+\zeta\|(I-T) x-(I-T) y\|^{2}, \\
\forall x, y \in C ;
\end{array}
$$

see [21] for more details. We denote by $\operatorname{Fix}(T)$ the fixed point set of $T$; that is, $\operatorname{Fix}(T)=\{x \in C: T x=x\}$.

We introduce and consider the following triple hierarchical variational inequality problem (THVIP).

Problem $I$. Let $F: C \rightarrow \mathscr{H}$ be $\kappa$-Lipschitzian and $\eta$-strongly monotone on the nonempty, closed, and convex subset $C$ of $\mathscr{H}$, where $\kappa$ and $\eta$ are positive constants. Let $V: C \rightarrow \mathscr{H}$ be a $\rho$-contraction with coefficient $\rho \in[0,1), S: C \rightarrow C$ be a nonexpansive mapping, and, for $i=1,2, T_{i}: C \rightarrow$ $C$ be $\zeta_{i}$-strictly pseudocontractive mapping with $\operatorname{Fix}\left(T_{1}\right) \cap$ $\operatorname{Fix}\left(T_{2}\right) \neq \emptyset$. Let $0<\mu<2 \eta / \kappa^{2}$ and $0<\gamma \leq \tau$, where $\tau=1-\sqrt{1-\mu\left(2 \eta-\mu \kappa^{2}\right)}$. Then the objective is to find $x^{*} \in \Xi$ such that

$$
\left\langle(\mu F-\gamma V) x^{*}, x-x^{*}\right\rangle \geq 0, \quad \forall x \in \Xi,
$$

where $\Xi$ denotes the solution set of the following hierarchical variational inequality problem (HVIP) of finding $z^{*} \in \operatorname{Fix}(T)$ such that

$$
\left\langle(\mu F-\gamma S) z^{*}, z-z^{*}\right\rangle \geq 0, \quad \forall z \in \operatorname{Fix}\left(T_{1}\right) \cap \operatorname{Fix}\left(T_{2}\right) .
$$

In particular, whenever $T_{1}=T$ a nonexpansive mapping, and $T_{2}=I$ an identity mapping, Problem $I$ reduces to the THVIP considered by Ceng et al. [22]. By combining the regularization method, the hybrid steepest-descent method, and the projection method, they proposed an iterative algorithm that generates a sequence via the explicit scheme and studied the convergence analysis of the sequences generated by the proposed method.

We consider and study the following triple hierarchical variational inequality problem.
Problem II. Let $F: C \rightarrow \mathscr{H}$ be $\kappa$-Lipschitzian and $\eta$-strongly monotone on the nonempty, closed, and convex subset $C$ of $\mathscr{H}$, where $\kappa$ and $\eta$ are positive constants. Let $A: C \rightarrow \mathscr{H}$ be a monotone and $L$-Lipschitzian mapping, $V: C \rightarrow \mathscr{H}$ be a $\rho$-contraction with coefficient $\rho \in[0,1), S: C \rightarrow C$ be a nonexpansive mapping, and $T: C \rightarrow C$ be a $\zeta$-strictly pseudocontractive mapping with $\operatorname{Fix}(T) \cap \Gamma \neq \emptyset$. Let $0<\mu<$ $2 \eta / \kappa^{2}$ and $0<\gamma \leq \tau$, where $\tau=1-\sqrt{1-\mu\left(2 \eta-\mu \kappa^{2}\right)}$. Then the objective is to find $x^{*} \in \Xi$ such that

$$
\left\langle(\mu F-\gamma V) x^{*}, x-x^{*}\right\rangle \geq 0, \quad \forall x \in \Xi,
$$

where $\Xi$ denotes the solution set of the following hierarchical variational inequality problem (HVIP) of finding $z^{*} \epsilon$ $\operatorname{Fix}(T) \cap \Gamma$ such that

$$
\left\langle(\mu F-\gamma S) z^{*}, z-z^{*}\right\rangle \geq 0, \quad \forall z \in \operatorname{Fix}(T) \cap \Gamma .
$$

We remark that Problem II is a generalization of Problem I. Indeed, in Problem II we put $T=T_{1}$ and $A=I-T_{2}$, where $T_{i}: C \rightarrow C$ is a $\zeta_{i}$-strictly pseudocontractive mapping for $i=$ 1,2 . Then from the definition of strictly pseudocontractive mapping, it follows that

$$
\begin{aligned}
& \left\langle T_{2} x-T_{2} y, x-y\right\rangle \\
& \leq\|x-y\|^{2}-\frac{1-\zeta_{2}}{2}\left\|\left(I-T_{2}\right) x-\left(I-T_{2}\right) y\right\|^{2}, \\
& \forall x, y \in C .
\end{aligned}
$$

It is clear that the mapping $A=I-T_{2}$ is $\left(1-\zeta_{2}\right) / 2$-inverse strongly monotone. Taking $L=2 /\left(1-\zeta_{2}\right)$, we know that $A$ : $C \rightarrow \mathscr{H}$ is a monotone and $L$-Lipschitzian mapping. In this case, $\Gamma=\operatorname{Fix}\left(T_{2}\right)$. Therefore, Problem II reduces to Problem I.

Motivated and inspired by Korpelevich's extragradient method [23] and the iterative method proposed in [22], we propose the following multistep hybrid extragradient method for solving Problem II.

Algorithm I. Let $F: C \rightarrow \mathscr{H}$ be $\kappa$-Lipschitzian and $\eta$ strongly monotone on the nonempty, closed, and convex subset $C$ of $\mathscr{H}, A: C \rightarrow \mathscr{H}$ be a monotone and $L$ Lipschitzian mapping, $V: C \rightarrow \mathscr{H}$ be a $\rho$-contraction with coefficient $\rho \in[0,1), S: C \rightarrow C$ be a nonexpansive mapping, and $T: C \rightarrow C$ be a $\zeta$-strictly pseudocontractive mapping. Let $\left\{\alpha_{n}\right\} \subset[0, \infty),\left\{v_{n}\right\} \subset(0,1 / L),\left\{\gamma_{n}\right\} \subset[0,1)$ and $\left\{\beta_{n}\right\},\left\{\delta_{n}\right\}$, $\left\{\sigma_{n}\right\},\left\{\lambda_{n}\right\} \subset(0,1), 0<\mu<2 \eta / \kappa^{2}$, and $0<\gamma \leq \tau$, where $\tau=1-\sqrt{1-\mu\left(2 \eta-\mu \kappa^{2}\right)}$. The sequence $\left\{x_{n}\right\}$ is generated by the following iterative scheme:

$$
\begin{gathered}
x_{0}=x \in C \text { chosen arbitrarily, } \\
y_{n}=P_{C}\left(x_{n}-v_{n} A_{n} x_{n}\right), \\
z_{n}=\beta_{n} x_{n}+\gamma_{n} P_{C}\left(x_{n}-v_{n} A_{n} y_{n}\right)+\sigma_{n} T P_{C}\left(x_{n}-v_{n} A_{n} y_{n}\right), \\
x_{n+1}=P_{C}\left[\lambda_{n} \gamma\left(\delta_{n} V x_{n}+\left(1-\delta_{n}\right) S x_{n}\right)+\left(I-\lambda_{n} \mu F\right) z_{n}\right], \\
\forall n \geq 0,
\end{gathered}
$$


where $A_{n}=\alpha_{n} I+A$ for all $n \geq 0$. In particular, if $V \equiv 0$, then (11) reduces to the following iterative scheme:

$$
\begin{gathered}
x_{0}=x \in C \text { chosen arbitrarily, } \\
y_{n}=P_{C}\left(x_{n}-v_{n} A_{n} x_{n}\right), \\
z_{n}=\beta_{n} x_{n}+\gamma_{n} P_{C}\left(x_{n}-v_{n} A_{n} y_{n}\right)+\sigma_{n} T P_{C}\left(x_{n}-v_{n} A_{n} y_{n}\right), \\
x_{n+1}=P_{C}\left[\lambda_{n}\left(1-\delta_{n}\right) \gamma S x_{n}+\left(I-\lambda_{n} \mu F\right) z_{n}\right], \quad \forall n \geq 0 .
\end{gathered}
$$

Further, if $S=V$, then (11) reduces to the following iterative scheme:

$$
\begin{gathered}
x_{0}=x \in C \text { chosen arbitrarily, } \\
y_{n}=P_{C}\left(x_{n}-v_{n} A_{n} x_{n}\right), \\
z_{n}=\beta_{n} x_{n}+\gamma_{n} P_{C}\left(x_{n}-v_{n} A_{n} y_{n}\right)+\sigma_{n} T P_{C}\left(x_{n}-v_{n} A_{n} y_{n}\right), \\
x_{n+1}=P_{C}\left[\lambda_{n} \gamma V x_{n}+\left(I-\lambda_{n} \mu F\right) z_{n}\right], \quad \forall n \geq 0
\end{gathered}
$$

moreover, if $S=V \equiv 0$, then (12) reduces to the following iterative scheme:

$$
\begin{gathered}
x_{0}=x \in C \text { chosen arbitrarily, } \\
y_{n}=P_{C}\left(x_{n}-v_{n} A_{n} x_{n}\right), \\
z_{n}=\beta_{n} x_{n}+\gamma_{n} P_{C}\left(x_{n}-v_{n} A_{n} y_{n}\right)+\sigma_{n} T P_{C}\left(x_{n}-v_{n} A_{n} y_{n}\right), \\
x_{n+1}=P_{C}\left[\left(I-\lambda_{n} \mu F\right) z_{n}\right], \quad \forall n \geq 0 .
\end{gathered}
$$

We prove that under appropriate conditions the sequence $\left\{x_{n}\right\}$ generated by Algorithm I converges strongly to a unique solution of Problem II. Our result improves and extends Theorem 4.1 in [22] in the following aspects.

(a) Problem II generalizes Problem I from the fixed point set $\operatorname{Fix}(T)$ of a nonexpansive mapping $T$ to the intersection $\operatorname{Fix}(T) \cap \Gamma$ of the fixed point set of a strictly pseudocontractive mapping $T$ and the solution set $\Gamma$ of VIP (2).

(b) The Korpelevich extragradient algorithm is extended to develop the multistep hybrid extragradient algorithm (i.e., Algorithm I) for solving Problem II by virtue of the iterative schemes in Theorem 4.1 in [22].

(c) The strong convergence of the sequence $\left\{x_{n}\right\}$ generated by Algorithm I holds under the lack of the same restrictions as those in Theorem 4.1 in [22].

(d) The boundedness requirement of the sequence $\left\{x_{n}\right\}$ in Theorem 4.1 in [22] is replaced by the boundedness requirement of the sequence $\left\{S x_{n}\right\}$.

We also consider and study the multistep hybrid extragradient algorithm (i.e., Algorithm I) for solving the following system of hierarchical variational inequalities (SHVI).
Problem III. Let $F: C \rightarrow \mathscr{H}$ be $\kappa$-Lipschitzian and $\eta$ strongly monotone on the nonempty, closed, and convex subset $C$ of $\mathscr{H}$, where $\kappa$ and $\eta$ are positive constants. Let $A: C \rightarrow \mathscr{H}$ be a monotone and $L$-Lipschitzian mapping, $V: C \rightarrow \mathscr{H}$ be a $\rho$-contraction with coefficient $\rho \in[0,1)$, $S: C \rightarrow C$ be a nonexpansive mapping, and $T: C \rightarrow C$ be a $\zeta$-strictly pseudocontractive mapping with $\operatorname{Fix}(T) \cap \Gamma \neq \emptyset$. Let $0<\mu<2 \eta / \kappa^{2}$ and $0<\gamma \leq \tau$, where $\tau=1-\sqrt{1-\mu\left(2 \eta-\mu \kappa^{2}\right)}$. Then the objective is to find $x^{*} \in \operatorname{Fix}(T) \cap \Gamma$ such that

$$
\begin{aligned}
& \left\langle(\mu F-\gamma V) x^{*}, x-x^{*}\right\rangle \geq 0, \quad \forall x \in \operatorname{Fix}(T) \cap \Gamma, \\
& \left\langle(\mu F-\gamma S) x^{*}, y-x^{*}\right\rangle \geq 0, \quad \forall y \in \operatorname{Fix}(T) \cap \Gamma .
\end{aligned}
$$

In particular, if $T=T_{1}$ and $A=I-T_{2}$ where $T_{i}: C \rightarrow$ $C$ is $\zeta_{i}$-strictly pseudocontractive for $i=1,2$, Problem III reduces to the following Problem IV.

Problem $I V$. Let $F: C \rightarrow \mathscr{H}$ be $\kappa$-Lipschitzian and $\eta$ strongly monotone on the nonempty, closed, and convex subset $C$ of $\mathscr{H}$, where $\kappa$ and $\eta$ are positive constants. Let $V: C \rightarrow \mathscr{H}$ be a $\rho$-contraction with coefficient $\rho \in[0,1)$, $S: C \rightarrow C$ be a nonexpansive mapping, and, for $i=1,2$, $T_{i}: C \rightarrow C$ be $\zeta_{i}$-strictly pseudocontractive mapping with $\operatorname{Fix}\left(T_{1}\right) \cap \operatorname{Fix}\left(T_{2}\right) \neq \emptyset$. Let $0<\mu<2 \eta / \kappa^{2}$ and $0<\gamma \leq \tau$, where $\tau=1-\sqrt{1-\mu\left(2 \eta-\mu \kappa^{2}\right)}$. Then the objective is to find $x^{*} \in \operatorname{Fix}\left(T_{1}\right) \cap \operatorname{Fix}\left(T_{2}\right)$ such that

$$
\begin{aligned}
& \left\langle(\mu F-\gamma V) x^{*}, x-x^{*}\right\rangle \geq 0, \quad \forall x \in \operatorname{Fix}\left(T_{1}\right) \cap \operatorname{Fix}\left(T_{2}\right), \\
& \left\langle(\mu F-\gamma S) x^{*}, y-x^{*}\right\rangle \geq 0, \quad \forall y \in \operatorname{Fix}\left(T_{1}\right) \cap \operatorname{Fix}\left(T_{2}\right) .
\end{aligned}
$$

We prove that under very mild conditions the sequence $\left\{x_{n}\right\}$ generated by Algorithm I converges strongly to a unique solution of Problem III.

\section{Preliminaries}

Let $C$ be a nonempty, closed, and convex subset of $\mathscr{H}$ and $V: C \rightarrow \mathscr{H}$ be a (possibly nonself) $\rho$-contraction mapping with coefficient $\rho \in[0,1)$; that is, there exists a constant $\rho \in$ $[0,1)$ such that $\|V x-V y\| \leq \rho\|x-y\|$, for all $x, y \in C$. Now we present some known results and definitions which will be used in the sequel.

The metric (or nearest point) projection from $\mathscr{H}$ onto $C$ is the mapping $P_{C}: \mathscr{H} \rightarrow C$ which assigns to each point $x \in \mathscr{H}$ the unique point $P_{C} x \in C$ satisfying the property

$$
\left\|x-P_{C} x\right\|=\inf _{y \in C}\|x-y\|=: d(x, C) .
$$

The following properties of projections are useful and pertinent to our purpose.

Proposition 1 (see [21]). Given any $x \in \mathscr{H}$ and $z \in C$. One has

(i) $z=P_{C} x \Leftrightarrow\langle x-z, y-z\rangle \leq 0$, for all $y \in C$, 
(ii) $z=P_{C} x \Leftrightarrow\|x-z\|^{2} \leq\|x-y\|^{2}-\|y-z\|^{2}$, for all $y \in C$;

(iii) $\left\langle P_{C} x-P_{C} y, x-y\right\rangle \geq\left\|P_{C} x-P_{C} y\right\|^{2}$, for all $x, y \in$ $\mathscr{H}$, which hence implies that $P_{C}$ is nonexpansive and monotone.

Definition 2. A mapping $T: \mathscr{H} \rightarrow \mathscr{H}$ is said to be

(a) nonexpansive if

$$
\|T x-T y\| \leq\|x-y\|, \quad \forall x, y \in \mathscr{H} ;
$$

(b) firmly nonexpansive if $2 T-I$ is nonexpansive, or, equivalently,

$$
\langle x-y, T x-T y\rangle \geq\|T x-T y\|^{2}, \quad \forall x, y \in \mathscr{H}
$$

alternatively, $T$ is firmly nonexpansive if and only if $T$ can be expressed as

$$
T=\frac{1}{2}(I+S)
$$

where $S: \mathscr{H} \rightarrow \mathscr{H}$ is nonexpansive; projections are firmly nonexpansive.

Definition 3. Let $T$ be a nonlinear operator whose domain is $D(T) \subseteq \mathscr{H}$ and whose range is $R(T) \subseteq \mathscr{H}$.

(a) $T$ is said to be monotone if

$$
\langle x-y, T x-T y\rangle \geq 0, \quad \forall x, y \in D(T) .
$$

(b) Given a number $\beta>0, T$ is said to be $\beta$-strongly monotone if

$$
\langle x-y, T x-T y\rangle \geq \beta\|x-y\|^{2}, \quad \forall x, y \in D(T) .
$$

(c) Given a number $v>0, T$ is said to be $v$-inverse strongly monotone $(\nu$-ism) if

$$
\langle x-y, T x-T y\rangle \geq v\|T x-T y\|^{2}, \quad \forall x, y \in D(T) .
$$

It can be easily seen that if $T$ is nonexpansive, then $I-T$ is monotone. It is also easy to see that a projection $P_{C}$ is 1 -ism.

Inverse strongly monotone (also referred to as cocoercive) operators have been applied widely in solving practical problems in various fields, for instance, in traffic assignment problems; see [24, 25].

Definition 4. A mapping $T: \mathscr{H} \rightarrow \mathscr{H}$ is said to be an averaged mapping if it can be written as the average of the identity $I$ and a nonexpansive mapping, that is,

$$
T \equiv(1-\alpha) I+\alpha S
$$

where $\alpha \in(0,1)$ and $S: \mathscr{H} \rightarrow \mathscr{H}$ are nonexpansive. More precisely, when the last equality holds, we say that $T$ is $\alpha$ averaged. Thus firmly nonexpansive mappings (in particular, projections) are 1/2-averaged maps.
Proposition 5 (see [26]). Let $T: \mathscr{H} \rightarrow \mathscr{H}$ be a given mapping.

(i) $T$ is nonexpansive if and only if the complement $I-T$ is $1 / 2$-ism.

(ii) If $T$ is $\nu$-ism, then, for $\gamma>0, \gamma T$ is $\nu / \gamma$-ism.

(iii) $T$ is averaged if and only if the complement $I-T$ is $\nu$-ism for some $v>1 / 2$. Indeed, for $\alpha \in(0,1), T$ is $\alpha$-averaged if and only if $I-T$ is $1 / 2 \alpha$-ism.

Proposition 6 (see $[26,27])$. Let $S, T, V: \mathscr{H} \rightarrow \mathscr{H}$ be given operators.

(i) If $T=(1-\alpha) S+\alpha V$ for some $\alpha \in(0,1)$ and if $S$ is averaged and $V$ is nonexpansive, then $T$ is averaged.

(ii) $T$ is firmly nonexpansive if and only if the complement $I-T$ is firmly nonexpansive.

(iii) If $T=(1-\alpha) S+\alpha V$ for some $\alpha \in(0,1)$ and if $S$ is firmly nonexpansive and $V$ is nonexpansive, then $T$ is averaged.

(iv) The composite of finitely many averaged mappings is averaged. That is, if each of the mappings $\left\{T_{i}\right\}_{i=1}^{N}$ is averaged, then so is the composite $T_{1} \circ \cdots \circ T_{N}$. In particular, if $T_{1}$ is $\alpha_{1}$-averaged and $T_{2}$ is $\alpha_{2}$-averaged, where $\alpha_{1}, \alpha_{2} \in(0,1)$, then the composite $T_{1} \circ T_{2}$ is $\alpha$ averaged, where $\alpha=\alpha_{1}+\alpha_{2}-\alpha_{1} \alpha_{2}$.

On the other hand, it is clear that, in a real Hilbert space $\mathscr{H}, T: C \rightarrow C$ is $\zeta$-strictly pseudocontractive if and only if there holds the following inequality:

$$
\begin{aligned}
\langle T x-T y, x-y\rangle \leq & \|x-y\|^{2} \\
& -\frac{1-\zeta}{2}\|(I-T) x-(I-T) y\|^{2}, \\
& \forall x, y \in C .
\end{aligned}
$$

This immediately implies that if $T$ is a $\zeta$-strictly pseudocontractive mapping, then $I-T$ is $(1-\zeta) / 2$-inverse strongly monotone; for further detail, we refer to [21] and the references therein. It is well known that the class of strict pseudocontractions strictly includes the class of nonexpansive mappings. The so-called demiclosedness principle for strict pseudocontractive mappings in the following lemma will often be used.

Lemma 7 (see [21, Proposition 2.1]). Let $C$ be a nonempty closed convex subset of a real Hilbert space $\mathscr{H}$ and $T: C \rightarrow C$ be a mapping.

(i) If $T$ is a $\zeta$-strictly pseudocontractive mapping, then $T$ satisfies the Lipschitz condition:

$$
\|T x-T y\| \leq \frac{1+\zeta}{1-\zeta}\|x-y\|, \quad \forall x, y \in C .
$$

(ii) If $T$ is a $\zeta$-strictly pseudocontractive mapping, then the mapping $I-T$ is semiclosed at 0 ; that is, if $\left\{x_{n}\right\}$ is a sequence in $C$ such that $x_{n} \rightarrow \tilde{x}$ and $(I-T) x_{n} \rightarrow 0$, then $(I-T) \tilde{x}=0$. 
(iii) If $T$ is a $\zeta$-(quasi-)strict pseudocontraction, then the fixed point set $\operatorname{Fix}(T)$ of $T$ is closed and convex so that the projection $P_{\mathrm{Fix}(T)}$ is well defined.

The following lemma plays a key role in proving strong convergence of the sequences generated by our algorithms.

Lemma 8 (see [28]). Let $\left\{a_{n}\right\}$ be a sequence of nonnegative real numbers satisfying the property:

$$
a_{n+1} \leq\left(1-s_{n}\right) a_{n}+s_{n} b_{n}+r_{n}, \quad n \geq 0,
$$

where $\left\{s_{n}\right\} \subset(0,1]$ and $\left\{b_{n}\right\}$ are such that

(i) $\sum_{n=0}^{\infty} s_{n}=\infty$;

(ii) either $\lim \sup _{n \rightarrow \infty} b_{n} \leq 0$ or $\sum_{n=0}^{\infty}\left|s_{n} b_{n}\right|<\infty$;

(iii) $\sum_{n=0}^{\infty} r_{n}<\infty$ where $r_{n} \geq 0$, for all $n \geq 0$.

Then, $\lim _{n \rightarrow \infty} a_{n}=0$.

The following lemma is not hard to prove.

Lemma 9 (see [20]). Let $V: C \rightarrow \mathscr{H}$ be a $\rho$-contraction with $\rho \in[0,1)$ and $T: C \rightarrow C$ be a nonexpansive mapping. Then

(i) $I-V$ is $(1-\rho)$-strongly monotone:

$$
\begin{array}{r}
\langle(I-V) x-(I-V) y, x-y\rangle \geq(1-\rho)\|x-y\|^{2}, \\
\forall x, y \in C ;
\end{array}
$$

(ii) $I-T$ is monotone:

$$
\langle(I-T) x-(I-T) y, x-y\rangle \geq 0, \quad \forall x, y \in C .
$$

The following lemma plays an important role in proving strong convergence of the sequences generated by our algorithm.

Lemma 10 (see [29]). Let $C$ be a nonempty closed convex subset of a real Hilbert space $\mathscr{H}$. Let $T: C \rightarrow C$ be a $\zeta$-strictly pseudo-contractive mapping. Let $\gamma$ and $\delta$ be two nonnegative real numbers such that $(\gamma+\delta) \zeta \leq \gamma$. Then

$$
\begin{array}{r}
\|\gamma(x-y)+\delta(T x-T y)\| \leq(\gamma+\delta)\|x-y\|, \\
\forall x, y \in C .
\end{array}
$$

Lemma 11 (see [30, Lemma 3.1]). Let $\lambda$ be a number in $(0,1]$ and let $\mu>0$. Let $F: C \rightarrow \mathscr{H}$ be an operator on $C$ such that, for some constants $\kappa, \eta>0, F$ is $\kappa$-Lipschitzian and $\eta$ strongly monotone, associating with a nonexpansive mapping $T: C \rightarrow C$, define the mapping $T^{\lambda}: C \rightarrow \mathscr{H}$ by

$$
T^{\lambda} x:=T x-\lambda \mu F(T x), \quad \forall x \in C
$$

Then $T^{\lambda}$ is a contraction provided that $\mu<2 \eta / \kappa^{2}$, that is,

$$
\left\|T^{\lambda} x-T^{\lambda} y\right\| \leq(1-\lambda \tau)\|x-y\|, \quad \forall x, y \in C,
$$

where $\tau=1-\sqrt{1-\mu\left(2 \eta-\mu \kappa^{2}\right)} \in(0,1]$. In particular, if $T$ is the identity mapping $I$, then

$$
\begin{array}{r}
\|(I-\lambda \mu F) x-(I-\lambda \mu F) y\| \leq(1-\lambda \tau)\|x-y\|, \\
\forall x, y \in C .
\end{array}
$$
[31].

The following lemma appears implicitly in Reineermann

Lemma 12. Let $\mathscr{H}$ be a Hilbert space. Then

$$
\begin{aligned}
\|\lambda x+(1-\lambda) y-z\|^{2} & \\
=\lambda\|x-z\|^{2}+(1-\lambda)\|y-z\|^{2} & \\
-\lambda(1-\lambda)\|x-y\|^{2}, \quad \forall x, y, z \in \mathscr{H}, & \forall \lambda \in[0,1] .
\end{aligned}
$$

The following lemma is not difficult to prove.

Lemma 13 (see [32]). Let $\left\{\alpha_{n}\right\}$ and $\left\{\beta_{n}\right\}$ be a sequence of nonnegative real numbers and a sequence of real numbers, respectively, such that $\lim \sup _{n \rightarrow \infty} \alpha_{n}<\infty$ and $\lim \sup _{n \rightarrow \infty} \beta_{n} \leq 0$. Then $\lim \sup _{n \rightarrow \infty} \alpha_{n} \beta_{n} \leq 0$.

A set-valued mapping $\widetilde{T}: H \rightarrow 2^{H}$ is called monotone if, for all $x, y \in H, f \in \widetilde{T} x$, and $g \in \widetilde{T} y$ imply $\langle x-y, f-$ $g\rangle \geq 0$. A monotone mapping $\widetilde{T}: H \rightarrow 2^{H}$ is maximal if its graph $G(\widetilde{T})$ is not properly contained in the graph of any other monotone mapping. It is known that a monotone mapping $\widetilde{T}$ is maximal if and only if, for $(x, f) \in H \times H,\langle x-y, f-g\rangle \geq 0$ for all $(y, g) \in G(\widetilde{T})$ implies $f \in \widetilde{T} x$. Let $A: C \rightarrow H$ be a monotone and $L$-Lipschitzian mapping, and let $N_{C} v$ be the normal cone to $C$ at $v \in C$, that is, $N_{C} v=\{w \in H:\langle v-$ $u, w\rangle \geq 0$, for all $u \in C\}$. Define

$$
\widetilde{T} v= \begin{cases}A v+N_{C} v, & \text { if } v \in C, \\ \emptyset, & \text { if } v \notin C .\end{cases}
$$

It is known that in this case $\widetilde{T}$ is maximal monotone, and $0 \epsilon$ $\widetilde{T} v$ if and only if $v \in \Gamma$; see [33].

\section{Main Results}

We are now in a position to present the convergence analysis of Algorithm I for solving Problem II.

Theorem 14. Let $F: C \rightarrow \mathscr{H}$ be a $\kappa$-Lipschitzian and $\eta$-strongly monotone operator with constants $\kappa, \eta>0$, respectively, $A: C \rightarrow \mathscr{H}$ be a $1 / L$-inverse strongly monotone mapping, $V: C \rightarrow \mathscr{H}$ be a $\rho$-contraction with coefficient $\rho \in[0,1), S: C \rightarrow C$ be a nonexpansive mapping, and $T: C \rightarrow C$ be a $\zeta$-strictly pseudocontractive mapping. Let $0<\mu<2 \eta / \kappa^{2}$ and $0<\gamma \leq \tau$, where $\tau=1-$ $\sqrt{1-\mu\left(2 \eta-\mu \kappa^{2}\right)}$. Assume that the solution set $\Xi$ of the HVIP (9) is nonempty and that the following conditions hold for 
the sequences $\left\{\alpha_{n}\right\} \subset[0, \infty),\left\{v_{n}\right\} \subset(0,1 / L),\left\{\gamma_{n}\right\} \subset[0,1)$ and $\left\{\beta_{n}\right\},\left\{\delta_{n}\right\},\left\{\sigma_{n}\right\},\left\{\lambda_{n}\right\} \subset(0,1)$ :

(C1) $\sum_{n=0}^{\infty} \alpha_{n}<\infty$ and $\lim _{n \rightarrow \infty}\left(\alpha_{n} / \lambda_{n}^{2}\right)=0$;

(C2) $0<\lim \inf _{n \rightarrow \infty} \nu_{n} \leq \lim \sup _{n \rightarrow \infty} \nu_{n}<1 / L$;

(C3) $\beta_{n}+\gamma_{n}+\sigma_{n}=1$ and $\left(\gamma_{n}+\sigma_{n}\right) \zeta \leq \gamma_{n}$ for all $n \geq 0$;

(C4) $0<\liminf _{n \rightarrow \infty} \beta_{n} \leq \lim \sup _{n \rightarrow \infty} \beta_{n}<1$ and

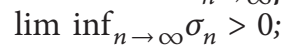

(C5) $\lim _{n \rightarrow \infty} \lambda_{n}=0, \lim _{n \rightarrow \infty} \delta_{n}=0$ and $\sum_{n=0}^{\infty} \delta_{n} \lambda_{n}=\infty$;

(C6) there are constants $\bar{k}, \theta>0$ satisfying $\|x-T x\| \geq$ $\bar{k}[d(x, \operatorname{Fix}(T) \cap \Gamma)]^{\theta}$ for each $x \in C$;

(C7) $\lim _{n \rightarrow \infty}\left(\lambda_{n}^{1 / \theta} / \delta_{n}\right)=0$.

One has the following.

(a) If $\left\{x_{n}\right\}$ is the sequence generated by scheme (11) and $\left\{S x_{n}\right\}$ is bounded, then $\left\{x_{n}\right\}$ converges strongly to the point $x^{*} \in \operatorname{Fix}(T) \cap \Gamma$ which is a unique solution of Problem II provided that $\left\|x_{n+1}-x_{n}\right\|+\left\|x_{n}-z_{n}\right\|=o\left(\lambda_{n}^{2}\right)$.

(b) If $\left\{x_{n}\right\}$ is the sequence generated by the scheme (12) and $\left\{S x_{n}\right\}$ is bounded, then $\left\{x_{n}\right\}$ converges strongly to a unique solution $x^{*}$ of the following VIP provided that $\left\|x_{n+1}-x_{n}\right\|+\left\|x_{n}-z_{n}\right\|=o\left(\lambda_{n}^{2}\right):$

find $x^{*} \in \Xi$ such that $\left\langle F x^{*}, x-x^{*}\right\rangle \geq 0, \quad \forall x \in \Xi$.

Proof. We treat only case (a); that is, the sequence $\left\{x_{n}\right\}$ is generated by the scheme (11). Obviously, from the condition $\Xi \neq \emptyset$ it follows that $\operatorname{Fix}(T) \cap \Gamma \neq \emptyset$. In addition, in terms of conditions (C2) and (C4), without loss of generality, we may assume that $\left\{v_{n}\right\} \subset[a, b]$ for some $a, b \in(0,1 / L),\left\{\beta_{n}\right\} \subset[c, d]$ for some $c, d \in(0,1)$.

First of all, we observe (see, e.g., [34]) that $P_{C}(I-\nu(\alpha I+A))$ and $P_{C}\left(I-v_{n} A_{n}\right)$ are nonexpansive for all $n \geq 0$.

Next we divide the remainder of the proof into several steps.

Step 1 ( $\left\{x_{n}\right\}$ is bounded). Indeed, take a fixed $p \in \operatorname{Fix}(T) \cap \Gamma$ arbitrarily. Then, we get $T p=p$ and $P_{C}(I-v A) p=p$ for $v \in(0,2 / L)$. From (11), it follows that

$$
\begin{aligned}
\left\|y_{n}-p\right\|= & \left\|P_{C}\left(I-v_{n} A_{n}\right) x_{n}-P_{C}\left(I-v_{n} A\right) p\right\| \\
\leq & \left\|P_{C}\left(I-v_{n} A_{n}\right) x_{n}-P_{C}\left(I-v_{n} A_{n}\right) p\right\| \\
& +\left\|P_{C}\left(I-v_{n} A_{n}\right) p-P_{C}\left(I-v_{n} A\right) p\right\| \\
\leq & \left\|x_{n}-p\right\|+\left\|\left(I-v_{n} A_{n}\right) p-\left(I-v_{n} A\right) p\right\| \\
= & \left\|x_{n}-p\right\|+v_{n} \alpha_{n}\|p\| .
\end{aligned}
$$

Put $t_{n}=P_{C}\left(x_{n}-v_{n} A_{n} y_{n}\right)$ for each $n \geq 0$. Then, by Proposition 1 (ii), we have

$$
\begin{aligned}
\left\|t_{n}-p\right\|^{2} \leq & \left\|x_{n}-v_{n} A_{n} y_{n}-p\right\|^{2}-\left\|x_{n}-v_{n} A_{n} y_{n}-t_{n}\right\|^{2} \\
= & \left\|x_{n}-p\right\|^{2}-\left\|x_{n}-t_{n}\right\|^{2}+2 v_{n}\left\langle A_{n} y_{n}, p-t_{n}\right\rangle \\
= & \left\|x_{n}-p\right\|^{2}-\left\|x_{n}-t_{n}\right\|^{2} \\
& +2 v_{n}\left(\left\langle A_{n} y_{n}-A_{n} p, p-y_{n}\right\rangle\right. \\
& \left.\quad+\left\langle A_{n} p, p-y_{n}\right\rangle+\left\langle A_{n} y_{n}, y_{n}-t_{n}\right\rangle\right) \\
\leq & \left\|x_{n}-p\right\|^{2}-\left\|x_{n}-t_{n}\right\|^{2} \\
& +2 v_{n}\left(\left\langle A_{n} p, p-y_{n}\right\rangle+\left\langle A_{n} y_{n}, y_{n}-t_{n}\right\rangle\right) \\
= & \left\|x_{n}-p\right\|^{2}-\left\|x_{n}-t_{n}\right\|^{2} \\
& +2 v_{n}\left[\left\langle\left(\alpha_{n} I+A\right) p, p-y_{n}\right\rangle+\left\langle A_{n} y_{n}, y_{n}-t_{n}\right\rangle\right] \\
\leq & \left\|x_{n}-p\right\|^{2}-\left\|x_{n}-t_{n}\right\|^{2} \\
& +2 v_{n}\left[\alpha_{n}\left\langle p, p-y_{n}\right\rangle+\left\langle A_{n} y_{n}, y_{n}-t_{n}\right\rangle\right] \\
= & \left\|x_{n}-p\right\|^{2}-\left\|x_{n}-y_{n}\right\|^{2} \\
& -2\left\langle x_{n}-y_{n}, y_{n}-t_{n}\right\rangle-\left\|y_{n}-t_{n}\right\|^{2} \\
& +2 v_{n}\left[\alpha_{n}\left\langle p, p-y_{n}\right\rangle+\left\langle A_{n} y_{n}, y_{n}-t_{n}\right\rangle\right] \\
= & \left\|x_{n}-p\right\|^{2}-\left\|x_{n}-y_{n}\right\|^{2}-\left\|y_{n}-t_{n}\right\|^{2} \\
& +2\left\langle x_{n}-v_{n} A_{n} y_{n}-y_{n}, t_{n}-y_{n}\right\rangle \\
& +2 v_{n} \alpha_{n}\left\langle p, p-y_{n}\right\rangle . \\
&
\end{aligned}
$$

Further, by Proposition 1 (i), we have

$$
\begin{aligned}
\left\langle x_{n}-v_{n} A_{n} y_{n}-y_{n}, t_{n}-y_{n}\right\rangle \\
=\left\langle x_{n}-v_{n} A_{n} x_{n}-y_{n}, t_{n}-y_{n}\right\rangle \\
\quad+\left\langle v_{n} A_{n} x_{n}-v_{n} A_{n} y_{n}, t_{n}-y_{n}\right\rangle \\
\leq\left\langle v_{n} A_{n} x_{n}-v_{n} A_{n} y_{n}, t_{n}-y_{n}\right\rangle \\
\leq v_{n}\left\|A_{n} x_{n}-A_{n} y_{n}\right\|\left\|t_{n}-y_{n}\right\| \\
\leq v_{n}\left(\alpha_{n}+L\right)\left\|x_{n}-y_{n}\right\|\left\|t_{n}-y_{n}\right\| .
\end{aligned}
$$

So, we obtain

$$
\begin{aligned}
\left\|t_{n}-p\right\|^{2} \leq & \left\|x_{n}-p\right\|^{2}-\left\|x_{n}-y_{n}\right\|^{2}-\left\|y_{n}-t_{n}\right\|^{2} \\
& +2\left\langle x_{n}-v_{n} A_{n} y_{n}-y_{n}, t_{n}-y_{n}\right\rangle \\
& +2 v_{n} \alpha_{n}\left\langle p, p-y_{n}\right\rangle \\
\leq & \left\|x_{n}-p\right\|^{2}-\left\|x_{n}-y_{n}\right\|^{2}-\left\|y_{n}-t_{n}\right\|^{2} \\
& +2 v_{n}\left(\alpha_{n}+L\right)\left\|x_{n}-y_{n}\right\|\left\|t_{n}-y_{n}\right\| \\
& +2 v_{n} \alpha_{n}\|p\|\left\|p-y_{n}\right\|
\end{aligned}
$$




$$
\begin{aligned}
\leq & \left\|x_{n}-p\right\|^{2}-\left\|x_{n}-y_{n}\right\|^{2}-\left\|y_{n}-t_{n}\right\|^{2} \\
& +v_{n}^{2}\left(\alpha_{n}+L\right)^{2}\left\|x_{n}-y_{n}\right\|^{2} \\
& +\left\|y_{n}-t_{n}\right\|^{2}+2 v_{n} \alpha_{n}\|p\|\left\|p-y_{n}\right\| \\
= & \left\|x_{n}-p\right\|^{2}+2 v_{n} \alpha_{n}\|p\|\left\|p-y_{n}\right\| \\
& +\left(v_{n}^{2}\left(\alpha_{n}+L\right)^{2}-1\right)\left\|x_{n}-y_{n}\right\|^{2} \\
\leq & \left\|x_{n}-p\right\|^{2}+2 v_{n} \alpha_{n}\|p\|\left\|p-y_{n}\right\| .
\end{aligned}
$$

Since $\left(\gamma_{n}+\sigma_{n}\right) \zeta \leq \gamma_{n}$, utilizing Lemmas 10 and 12, from (37) and the last inequality, we conclude that

$$
\begin{aligned}
& \left\|z_{n}-p\right\|^{2}=\left\|\beta_{n} x_{n}+\gamma_{n} t_{n}+\sigma_{n} T t_{n}-p\right\|^{2} \\
& =\| \beta_{n}\left(x_{n}-p\right)+\left(\gamma_{n}+\sigma_{n}\right) \frac{1}{\gamma_{n}+\sigma_{n}} \\
& \times\left[\gamma_{n}\left(t_{n}-p\right)+\sigma_{n}\left(T t_{n}-p\right)\right] \|^{2} \\
& =\beta_{n}\left\|x_{n}-p\right\|^{2}+\left(\gamma_{n}+\sigma_{n}\right) \\
& \times\left\|\frac{1}{\gamma_{n}+\sigma_{n}}\left[\gamma_{n}\left(t_{n}-p\right)+\sigma_{n}\left(T t_{n}-p\right)\right]\right\|^{2} \\
& -\beta_{n}\left(\gamma_{n}+\sigma_{n}\right) \\
& \times\left\|\frac{1}{\gamma_{n}+\sigma_{n}}\left[\gamma_{n}\left(t_{n}-x_{n}\right)+\sigma_{n}\left(T t_{n}-x_{n}\right)\right]\right\|^{2} \\
& \leq \beta_{n}\left\|x_{n}-p\right\|^{2}+\left(1-\beta_{n}\right)\left\|t_{n}-p\right\|^{2} \\
& -\frac{\beta_{n}}{1-\beta_{n}}\left\|z_{n}-x_{n}\right\|^{2} \\
& \leq \beta_{n}\left\|x_{n}-p\right\|^{2}+\left(1-\beta_{n}\right) \\
& \times\left[\left\|x_{n}-p\right\|^{2}+2 v_{n} \alpha_{n}\|p\|\left\|p-y_{n}\right\|\right. \\
& \left.+\left(v_{n}^{2}\left(\alpha_{n}+L\right)^{2}-1\right)\left\|x_{n}-y_{n}\right\|^{2}\right] \\
& -\frac{\beta_{n}}{1-\beta_{n}}\left\|z_{n}-x_{n}\right\|^{2} \\
& \leq\left\|x_{n}-p\right\|^{2}+2 v_{n} \alpha_{n}\|p\|\left\|p-y_{n}\right\| \\
& +\left(1-\beta_{n}\right)\left(v_{n}^{2}\left(\alpha_{n}+L\right)^{2}-1\right)\left\|x_{n}-y_{n}\right\|^{2} \\
& -\frac{\beta_{n}}{1-\beta_{n}}\left\|z_{n}-x_{n}\right\|^{2}
\end{aligned}
$$

$$
\begin{aligned}
\leq & \left\|x_{n}-p\right\|^{2}+2 v_{n} \alpha_{n}\|p\|\left(\left\|x_{n}-p\right\|+v_{n} \alpha_{n}\|p\|\right) \\
& +\left(1-\beta_{n}\right)\left(v_{n}^{2}\left(\alpha_{n}+L\right)^{2}-1\right)\left\|x_{n}-y_{n}\right\|^{2} \\
& -\frac{\beta_{n}}{1-\beta_{n}}\left\|z_{n}-x_{n}\right\|^{2} \\
\leq & \left\|x_{n}-p\right\|^{2}+2\left\|x_{n}-p\right\|\left(\sqrt{2} v_{n} \alpha_{n}\|p\|\right) \\
& +\left(\sqrt{2} v_{n} \alpha_{n}\|p\|\right)^{2} \\
& +\left(1-\beta_{n}\right)\left(v_{n}^{2}\left(\alpha_{n}+L\right)^{2}-1\right)\left\|x_{n}-y_{n}\right\|^{2} \\
& -\frac{\beta_{n}}{1-\beta_{n}}\left\|z_{n}-x_{n}\right\|^{2} \\
= & \left(\left\|x_{n}-p\right\|+\sqrt{2} v_{n} \alpha_{n}\|p\|\right)^{2} \\
& +\left(1-\beta_{n}\right)\left(v_{n}^{2}\left(\alpha_{n}+L\right)^{2}-1\right)\left\|x_{n}-y_{n}\right\|^{2} \\
& -\frac{\beta_{n}}{1-\beta_{n}}\left\|z_{n}-x_{n}\right\|^{2} \\
\leq & \left(\left\|x_{n}-p\right\|+\sqrt{2} v_{n} \alpha_{n}\|p\|\right)^{2} .
\end{aligned}
$$

Noticing the boundedness of $\left\{S x_{n}\right\}$, we get $\sup _{n \geq 0} \| \gamma S x_{n}-$ $\mu F p \| \leq M$ for some $M \geq 0$. Moreover, utilizing Lemma 11 we have from (11)

$$
\begin{aligned}
\left\|x_{n+1}-p\right\| & \\
= & \| P_{C}\left[\lambda_{n} \gamma\left(\delta_{n} V x_{n}+\left(1-\delta_{n}\right) S x_{n}\right)\right. \\
& \left.\quad+\left(I-\lambda_{n} \mu F\right) z_{n}\right]-P_{C} p \| \\
\leq & \left\|\lambda_{n} \gamma\left(\delta_{n} V x_{n}+\left(1-\delta_{n}\right) S x_{n}\right)+\left(I-\lambda_{n} \mu F\right) z_{n}-p\right\| \\
= & \| \lambda_{n} \gamma\left(\delta_{n} V x_{n}+\left(1-\delta_{n}\right) S x_{n}\right) \\
& \quad-\lambda_{n} \mu F p+\left(I-\lambda_{n} \mu F\right) z_{n}-\left(I-\lambda_{n} \mu F\right) p \| \\
\leq & \left\|\lambda_{n} \gamma\left(\delta_{n} V x_{n}+\left(1-\delta_{n}\right) S x_{n}\right)-\lambda_{n} \mu F p\right\| \\
& +\left\|\left(I-\lambda_{n} \mu F\right) z_{n}-\left(I-\lambda_{n} \mu F\right) p\right\| \\
= & \lambda_{n}\left\|\delta_{n}\left(\gamma V x_{n}-\mu F p\right)+\left(1-\delta_{n}\right)\left(\gamma S x_{n}-\mu F p\right)\right\| \\
& +\left\|\left(I-\lambda_{n} \mu F\right) z_{n}-\left(I-\lambda_{n} \mu F\right) p\right\| \\
\leq & \lambda_{n} \delta_{n}\left\|\gamma V x_{n}-\mu F p\right\| \\
& +\left(1-\delta_{n}\right)\left\|\gamma S x_{n}-\mu F p\right\| \\
& +\left(1-\lambda_{n} \tau\right)\left\|z_{n}-p\right\| \\
\leq & \lambda_{n}\left[\delta_{n}\left(\left\|\gamma V x_{n}-\gamma V p\right\|+\|\gamma V p-\mu F p\|\right)+\left(1-\delta_{n}\right) M\right] \\
& +\left(1-\lambda_{n} \tau\right)\left\|z_{n}-p\right\|
\end{aligned}
$$




$$
\begin{aligned}
\leq & \lambda_{n}\left[\delta_{n} \gamma \rho\left\|x_{n}-p\right\|+\delta_{n}\|\gamma V p-\mu F p\|+\left(1-\delta_{n}\right) M\right] \\
& +\left(1-\lambda_{n} \tau\right)\left[\left\|x_{n}-p\right\|+\sqrt{2} v_{n} \alpha_{n}\|p\|\right] \\
\leq & \lambda_{n}\left[\delta_{n} \gamma \rho\left\|x_{n}-p\right\|+\max \{M,\|\gamma V p-\mu F p\|\}\right] \\
& +\left(1-\lambda_{n} \tau\right)\left[\left\|x_{n}-p\right\|+\sqrt{2} v_{n} \alpha_{n}\|p\|\right] \\
\leq & \lambda_{n} \gamma \rho\left\|x_{n}-p\right\|+\lambda_{n} \max \{M,\|\gamma V p-\mu F p\|\} \\
& +\left(1-\lambda_{n} \tau\right)\left\|x_{n}-p\right\|+\sqrt{2} v_{n} \alpha_{n}\|p\| \\
= & {\left[1-(\tau-\gamma \rho) \lambda_{n}\right]\left\|x_{n}-p\right\| } \\
& +\lambda_{n} \max \{M,\|\gamma V p-\mu F p\|\}+\sqrt{2} v_{n} \alpha_{n}\|p\| .
\end{aligned}
$$

So, calling

$$
\widetilde{M}=\max \left\{\left\|x_{0}-p\right\|, \frac{M}{\tau-\gamma \rho}, \frac{\|\gamma V p-\mu F p\|}{\tau-\gamma \rho}\right\},
$$

we claim that

$$
\left\|x_{n+1}-p\right\| \leq \widetilde{M}+\sum_{j=0}^{n} \sqrt{2} v_{j} \alpha_{j}\|p\|, \quad \forall n \geq 0 .
$$

Indeed, when $n=0$, it is clear from (42) that (44) is valid, that is,

$$
\left\|x_{1}-p\right\| \leq \widetilde{M}+\sum_{j=0}^{0} \sqrt{2} v_{j} \alpha_{j}\|p\| .
$$

Assume that (44) is valid for $n(\geq 1)$, that is,

$$
\left\|x_{n}-p\right\| \leq \widetilde{M}+\sum_{j=0}^{n-1} \sqrt{2} v_{j} \alpha_{j}\|p\| .
$$

Then from (42) and (46) it follows that

$$
\begin{aligned}
\left\|x_{n+1}-p\right\| \leq & {\left[1-(\tau-\gamma \rho) \lambda_{n}\right]\left\|x_{n}-p\right\| } \\
& +\lambda_{n} \max \{M,\|\gamma V p-\mu F p\|\}+\sqrt{2} v_{n} \alpha_{n}\|p\| \\
\leq & {\left[1-(\tau-\gamma \rho) \lambda_{n}\right]\left[\widetilde{M}+\sum_{j=0}^{n-1} \sqrt{2} v_{j} \alpha_{j}\|p\|\right] } \\
& +\lambda_{n} \max \{M,\|\gamma V p-\mu F p\|\}+\sqrt{2} v_{n} \alpha_{n}\|p\| \\
\leq & {\left[1-(\tau-\gamma \rho) \lambda_{n}\right] \widetilde{M}+\sum_{j=0}^{n-1} \sqrt{2} v_{j} \alpha_{j}\|p\| } \\
& +(\tau-\gamma \rho) \lambda_{n} \max \left\{\frac{M}{\tau-\gamma \rho}, \frac{\|\gamma V p-\mu F p\|}{\tau-\gamma \rho}\right\} \\
& +\sqrt{2} v_{n} \alpha_{n}\|p\| \\
\leq & \widetilde{M}+\sum_{j=0}^{n} \sqrt{2} v_{j} \alpha_{j}\|p\| .
\end{aligned}
$$

This shows that (44) is also valid for $n+1$. Hence, by induction we derive the claim. Consequently, $\left\{x_{n}\right\}$ is bounded (due to $\left.\sum_{n=0}^{\infty} \alpha_{n}<\infty\right)$ and so are $\left\{y_{n}\right\},\left\{z_{n}\right\},\left\{A x_{n}\right\}$, and $\left\{A y_{n}\right\}$.

Step $2\left(\lim _{n \rightarrow \infty}\left\|x_{n}-y_{n}\right\|=\lim _{n \rightarrow \infty}\left\|x_{n}-t_{n}\right\|=\lim _{n \rightarrow \infty} \| t_{n}-\right.$ $\left.T t_{n} \|=0\right)$. Indeed, from (11) and (41), it follows that

$$
\begin{aligned}
&\left\|x_{n+1}-p\right\|^{2} \\
& \leq\left\|\lambda_{n} \gamma\left(\delta_{n} V x_{n}+\left(1-\delta_{n}\right) S x_{n}\right)+\left(I-\lambda_{n} \mu F\right) z_{n}-p\right\|^{2} \\
&= \| \lambda_{n} \gamma\left(\delta_{n} V x_{n}+\left(1-\delta_{n}\right) S x_{n}\right) \\
&-\lambda_{n} \mu F p+\left(I-\lambda_{n} \mu F\right) z_{n}-\left(I-\lambda_{n} \mu F\right) p \|^{2} \\
& \leq\left\{\left\|\lambda_{n} \gamma\left(\delta_{n} V x_{n}+\left(1-\delta_{n}\right) S x_{n}\right)-\lambda_{n} \mu F p\right\|\right. \\
&\left.+\left\|\left(I-\lambda_{n} \mu F\right) z_{n}-\left(I-\lambda_{n} \mu F\right) p\right\|\right\}^{2} \\
& \leq\left\{\lambda_{n}\left\|\delta_{n}\left(\gamma V x_{n}-\mu F p\right)+\left(1-\delta_{n}\right)\left(\gamma S x_{n}-\mu F p\right)\right\|\right. \\
&\left.+\left(1-\lambda_{n} \tau\right)\left\|z_{n}-p\right\|\right\}^{2} \\
& \leq \lambda_{n} \frac{1}{\tau}\left[\delta_{n}\left\|\gamma V x_{n}-\mu F p\right\|+\left(1-\delta_{n}\right)\left\|\gamma S x_{n}-\mu F p\right\|\right]^{2} \\
&+\left(1-\lambda_{n} \tau\right)\left\|z_{n}-p\right\|^{2} \\
& \leq \lambda_{n} \frac{1}{\tau}\left[\left\|\gamma V x_{n}-\mu F p\right\|+\left\|\gamma S x_{n}-\mu F p\right\|\right]^{2}+\left\|z_{n}-p\right\|^{2} \\
& \leq \lambda_{n} \frac{1}{\tau}\left[\left\|\gamma V x_{n}-\mu F p\right\|+\left\|\gamma S x_{n}-\mu F p\right\|\right]^{2} \\
&+\left(\left\|x_{n}-p\right\|+\sqrt{2} v_{n} \alpha_{n}\|p\|\right)^{2} \\
&+\left(1-\beta_{n}\right)\left(v_{n}^{2}\left(\alpha_{n}+L\right)^{2}-1\right)\left\|x_{n}-y_{n}\right\|^{2} \\
& \leq\left(\left\|x_{n}-p\right\|+\sqrt{2} v_{n} \alpha_{n}\|p\|\right)^{2}+\lambda_{n} M_{1} \\
&+\left(1-\beta_{n}\right)\left(v_{n}^{2}\left(\alpha_{n}+L\right)^{2}-1\right)\left\|x_{n}-y_{n}\right\|^{2} \\
&-\frac{\beta_{n}}{1-\beta_{n}}\left\|z_{n}-x_{n}\right\|^{2} \\
&
\end{aligned}
$$

where $M_{1}=\sup _{n \geq 0}\left\{(1 / \tau)\left[\left\|\gamma V x_{n}-\mu F p\right\|+\left\|\gamma S x_{n}-\mu F p\right\|\right]^{2}\right\}$. This together with $\left\{v_{n}\right\} \subset[a, b] \subset(0,1 / L)$ and $\left\{\beta_{n}\right\} \subset[c, d] \subset$ $(0,1)$ implies that

$$
\begin{aligned}
(1-d) & \left(1-b^{2}\left(\alpha_{n}+L\right)^{2}\right)\left\|x_{n}-y_{n}\right\|^{2}+\frac{c}{1-c}\left\|z_{n}-x_{n}\right\|^{2} \\
\leq & \left(1-\beta_{n}\right)\left(1-v_{n}^{2}\left(\alpha_{n}+L\right)^{2}\right)\left\|x_{n}-y_{n}\right\|^{2} \\
& +\frac{\beta_{n}}{1-\beta_{n}}\left\|z_{n}-x_{n}\right\|^{2}
\end{aligned}
$$




$$
\begin{aligned}
\leq & \left(\left\|x_{n}-p\right\|+\sqrt{2} v_{n} \alpha_{n}\|p\|\right)^{2}-\left\|x_{n+1}-p\right\|^{2}+\lambda_{n} M_{1} \\
= & {\left[\left(\left\|x_{n}-p\right\|+\sqrt{2} v_{n} \alpha_{n}\|p\|\right)-\left\|x_{n+1}-p\right\|\right] } \\
& \times\left[\left(\left\|x_{n}-p\right\|+\sqrt{2} v_{n} \alpha_{n}\|p\|\right)+\left\|x_{n+1}-p\right\|\right]+\lambda_{n} M_{1} \\
\leq & {\left[\left\|x_{n+1}-x_{n}\right\|+\sqrt{2} v_{n} \alpha_{n}\|p\|\right] } \\
& \times\left[\left\|x_{n}-p\right\|+\left\|x_{n+1}-p\right\|+\sqrt{2} v_{n} \alpha_{n}\|p\|\right]+\lambda_{n} M_{1} \\
\leq & {\left[\left\|x_{n+1}-x_{n}\right\|+\sqrt{2} b \alpha_{n}\|p\|\right] } \\
& \times\left[\left\|x_{n}-p\right\|+\left\|x_{n+1}-p\right\|+\sqrt{2} b \alpha_{n}\|p\|\right]+\lambda_{n} M_{1} .
\end{aligned}
$$

Note that $\lim _{n \rightarrow \infty} \alpha_{n}=\lim _{n \rightarrow \infty} \lambda_{n}=0$. Hence, taking into account the boundedness of $\left\{x_{n}\right\}$ and $\lim _{n \rightarrow \infty}\left\|x_{n+1}-x_{n}\right\|=0$, we deduce from (49) that

$$
\lim _{n \rightarrow \infty}\left\|x_{n}-y_{n}\right\|=\lim _{n \rightarrow \infty}\left\|z_{n}-x_{n}\right\|=0 .
$$

Furthermore, we obtain

$$
\begin{aligned}
\left\|y_{n}-t_{n}\right\| & =\left\|P_{C}\left(x_{n}-v_{n} A_{n} x_{n}\right)-P_{C}\left(x_{n}-v_{n} A_{n} y_{n}\right)\right\| \\
& \leq\left\|\left(x_{n}-v_{n} A_{n} x_{n}\right)-\left(x_{n}-v_{n} A_{n} y_{n}\right)\right\| \\
& =v_{n}\left\|A_{n} x_{n}-A_{n} y_{n}\right\| \\
& \leq v_{n}\left(\alpha_{n}+L\right)\left\|x_{n}-y_{n}\right\|,
\end{aligned}
$$

which together with (50) implies that

$$
\lim _{n \rightarrow \infty}\left\|y_{n}-t_{n}\right\|=0, \quad \lim _{n \rightarrow \infty}\left\|x_{n}-t_{n}\right\|=0 .
$$

So, from (11) we get

$$
\begin{aligned}
\left\|\sigma_{n}\left(T t_{n}-x_{n}\right)\right\| & =\left\|z_{n}-x_{n}-\gamma_{n}\left(t_{n}-x_{n}\right)\right\| \\
& \leq\left\|z_{n}-x_{n}\right\|+\gamma_{n}\left\|t_{n}-x_{n}\right\| \longrightarrow 0,
\end{aligned}
$$

which together with $\liminf _{n \rightarrow \infty} \sigma_{n}>0$ implies that

$$
\lim _{n \rightarrow \infty}\left\|T t_{n}-x_{n}\right\|=0 \text {. }
$$

Note that

$$
\left\|t_{n}-T t_{n}\right\| \leq\left\|t_{n}-y_{n}\right\|+\left\|y_{n}-x_{n}\right\|+\left\|x_{n}-T t_{n}\right\| .
$$

This together with (50)-(54) implies that

$$
\lim _{n \rightarrow \infty}\left\|t_{n}-T t_{n}\right\|=0 .
$$

Step $3\left(\omega_{w}\left(x_{n}\right) \subset \operatorname{Fix}(T) \cap \Gamma\right)$. Indeed, since $A$ is $L$-Lipschitz continuous, we have

$$
\lim _{n \rightarrow \infty}\left\|A y_{n}-A t_{n}\right\|=0 .
$$

As $\left\{x_{n}\right\}$ is bounded, there is a subsequence $\left\{x_{n_{n}}\right\}$ of $\left\{x_{n}\right\}$ that converges weakly to some $\hat{x}$. By the same argument as that in [34], we can obtain that $\widehat{x} \in \operatorname{Fix}(T) \cap \Gamma$ from which it follows that

$$
\omega_{w}\left(x_{n}\right) \subset \operatorname{Fix}(T) \cap \Gamma .
$$

Step $4\left(\omega_{w}\left(x_{n}\right) \subset \Xi\right)$. Indeed, we first note that $0<\gamma \leq \tau$ and

$$
\begin{aligned}
\mu \eta \geq \tau & \Longleftrightarrow \mu \eta \geq 1-\sqrt{1-\mu\left(2 \eta-\mu \kappa^{2}\right)} \\
& \Longleftrightarrow \sqrt{1-\mu\left(2 \eta-\mu \kappa^{2}\right)} \geq 1-\mu \eta \\
& \Longleftrightarrow 1-2 \mu \eta+\mu^{2} \kappa^{2} \geq 1-2 \mu \eta+\mu^{2} \eta^{2} \\
& \Longleftrightarrow \kappa^{2} \geq \eta^{2} \\
& \Longleftrightarrow \kappa \geq \eta .
\end{aligned}
$$

It is clear that

$$
\begin{aligned}
& \langle(\mu F-\gamma S) x-(\mu F-\gamma S) y, x-y\rangle \\
& \quad \geq(\mu \eta-\gamma)\|x-y\|^{2}, \quad \forall x, y \in C .
\end{aligned}
$$

Hence, it follows from $0<\gamma \leq \tau \leq \mu \eta$ that $\mu F-\gamma S$ is monotone. Putting

$$
\begin{array}{r}
w_{n}=\lambda_{n} \gamma\left(\delta_{n} V x_{n}+\left(1-\delta_{n}\right) S x_{n}\right)+\left(\mathrm{I}-\lambda_{n} \mu F\right) T z_{n}, \\
\forall n \geq 0,
\end{array}
$$

and noticing from (11)

$$
\begin{aligned}
x_{n+1}= & P_{C} w_{n}-w_{n}+\lambda_{n} \gamma\left(\delta_{n} V x_{n}+\left(1-\delta_{n}\right) S x_{n}\right) \\
& +\left(I-\lambda_{n} \mu F\right) z_{n},
\end{aligned}
$$

we obtain

$$
\begin{aligned}
x_{n}-x_{n+1}= & w_{n}-P_{C} w_{n}+\delta_{n} \lambda_{n}(\mu F-\gamma V) x_{n} \\
& +\lambda_{n}\left(1-\delta_{n}\right)(\mu F-\gamma S) x_{n}+\left(I-\lambda_{n} \mu F\right) x_{n} \\
& -\left(I-\lambda_{n} \mu F\right) z_{n} .
\end{aligned}
$$

Set

$$
e_{n}=\frac{x_{n}-x_{n+1}}{\lambda_{n}\left(1-\delta_{n}\right)}, \quad \forall n \geq 0 .
$$

It can be easily seen from (63) that

$$
\begin{aligned}
e_{n}= & \frac{w_{n}-P_{C} w_{n}}{\lambda_{n}\left(1-\delta_{n}\right)}+(\mu F-\gamma S) x_{n} \\
& +\frac{\delta_{n}}{1-\delta_{n}}(\mu F-\gamma V) x_{n} \\
& +\frac{\left(I-\lambda_{n} \mu F\right) x_{n}-\left(I-\lambda_{n} \mu F\right) z_{n}}{\lambda_{n}\left(1-\delta_{n}\right)} .
\end{aligned}
$$


This yields that, for all $w \in \operatorname{Fix}(T) \cap \Gamma$ (noticing $\left.x_{n}=P_{C} w_{n-1}\right)$,

$$
\begin{aligned}
\left\langle e_{n}, x_{n}-w\right\rangle= & \frac{1}{\lambda_{n}\left(1-\delta_{n}\right)}\left\langle w_{n}-P_{C} w_{n}, P_{C} w_{n-1}-w\right\rangle \\
& +\left\langle(\mu F-\gamma S) x_{n}, x_{n}-w\right\rangle \\
& +\frac{\delta_{n}}{1-\delta_{n}}\left\langle(\mu F-\gamma V) x_{n}, x_{n}-w\right\rangle \\
& +\frac{1}{\lambda_{n}\left(1-\delta_{n}\right)}\left\langle\left(I-\lambda_{n} \mu F\right) x_{n}\right. \\
= & \frac{1}{\lambda_{n}\left(1-\delta_{n}\right)}\left\langle w_{n}-P_{C} w_{n}, P_{C} w_{n}-w\right\rangle \\
& +\frac{1}{\lambda_{n}\left(1-\delta_{n}\right)}\left\langle w_{n}-P_{C} w_{n}, P_{C} w_{n-1}-P_{C} w_{n}\right\rangle \\
& +\left\langle(\mu F-\gamma S) w, x_{n}-w\right\rangle \\
& +\left\langle(\mu F-\gamma S) x_{n}-(\mu F-\gamma S) w, x_{n}-w\right\rangle \\
& +\frac{\delta_{n}}{1-\delta_{n}}\left\langle(\mu F-\gamma V) x_{n}, x_{n}-w\right\rangle \\
& +\frac{1}{\lambda_{n}\left(1-\delta_{n}\right)}\left\langle\left(I-\lambda_{n} \mu F\right) x_{n}\right. \\
& \left.-\left(I-\lambda_{n} \mu F\right) z_{n}, x_{n}-w\right\rangle . \\
& \\
& \\
&
\end{aligned}
$$

In (66), the first term is nonnegative due to Proposition 1, and the fourth term is also nonnegative due to the monotonicity of $\mu F-\gamma S$. We, therefore, deduce from (66) that (noticing again $\left.x_{n+1}=P_{C} w_{n}\right)$

$$
\begin{aligned}
\left\langle e_{n}, x_{n}-w\right\rangle \geq & \frac{1}{\lambda_{n}\left(1-\delta_{n}\right)}\left\langle w_{n}-P_{C} w_{n}, P_{C} w_{n-1}-P_{C} w_{n}\right\rangle \\
& +\left\langle(\mu F-\gamma S) w, x_{n}-w\right\rangle \\
& +\frac{\delta_{n}}{1-\delta_{n}}\left\langle(\mu F-\gamma V) x_{n}, x_{n}-w\right\rangle \\
& +\frac{1}{\lambda_{n}\left(1-\delta_{n}\right)}\left\langle\left(I-\lambda_{n} \mu F\right) x_{n}\right. \\
= & \left\langle w_{n}-P_{C} w_{n}, e_{n}\right\rangle+\left\langle(\mu F-\gamma S) w, x_{n}-w\right\rangle \\
& +\frac{\delta_{n}}{1-\delta_{n}}\left\langle(\mu F-\gamma V) x_{n}, x_{n}-w\right\rangle \\
& +\frac{1}{\lambda_{n}\left(1-\delta_{n}\right)}\left\langle\left(I-\lambda_{n} \mu F\right) x_{n}\right. \\
& \left.-\left(I-\lambda_{n} \mu F\right) z_{n}, x_{n}-w\right\rangle .
\end{aligned}
$$

Note that

$$
\left\|\left(I-\lambda_{n} \mu F\right) x_{n}-\left(I-\lambda_{n} \mu F\right) z_{n}\right\| \leq\left(1-\lambda_{n} \tau\right)\left\|x_{n}-z_{n}\right\| .
$$

Hence it follows from $\left\|x_{n}-z_{n}\right\|=o\left(\lambda_{n}\right)$ that

$$
\frac{\left\|\left(I-\lambda_{n} \mu F\right) x_{n}-\left(I-\lambda_{n} \mu F\right) z_{n}\right\|}{\lambda_{n}} \longrightarrow 0 .
$$

Also, since $e_{n} \rightarrow 0$ (due to $\left.\left\|x_{n+1}-x_{n}\right\|=o\left(\lambda_{n}\right)\right), \delta_{n} \rightarrow 0$ and $\left\{x_{n}\right\}$ is bounded by Step 1 which implies that $\left\{w_{n}\right\}$ is bounded, we obtain from (67) that

$$
\limsup _{n \rightarrow \infty}\left\langle(\mu F-\gamma S) w, x_{n}-w\right\rangle \leq 0, \quad \forall w \in \operatorname{Fix}(T) \cap \Gamma .
$$

This suffices to guarantee that $\omega_{w}\left(x_{n}\right) \subset \Xi$; namely, every weak limit point of $\left\{x_{n}\right\}$ solves the HVIP (9). As a matter of fact, if $x_{n_{i}} \rightarrow \tilde{x} \in \omega_{w}\left(x_{n}\right)$ for some subsequence $\left\{x_{n_{i}}\right\}$ of $\left\{x_{n}\right\}$, then we deduce from (70) that

$$
\begin{array}{r}
\langle(\mu F-\gamma S) w, \tilde{x}-w\rangle \\
\leq \limsup _{n \rightarrow \infty}\left\langle(\mu F-\gamma S) w, x_{n}-w\right\rangle \leq 0, \\
\forall w \in \operatorname{Fix}(T) \cap \Gamma,
\end{array}
$$

that is,

$$
\langle(\mu F-\gamma S) w, w-\tilde{x}\rangle \geq 0, \quad \forall w \in \operatorname{Fix}(T) \cap \Gamma .
$$

In addition, note that $\omega_{w}\left(x_{n}\right) \subset \operatorname{Fix}(T) \cap \Gamma$ by Step 3. Since $\mu F-\gamma S$ is monotone and Lipschitz continuous and $\operatorname{Fix}(T) \cap \Gamma$ is nonempty, closed, and convex, by the Minty lemma [1] the last inequality is equivalent to (9). Thus, we get $\tilde{x} \in \Xi$.

Step $5\left(\left\{x_{n}\right\}\right.$ converges strongly to a unique solution $x^{*}$ of Problem II.) Indeed, we now take a subsequence $\left\{x_{n_{i}}\right\}$ of $\left\{x_{n}\right\}$ satisfying

$$
\begin{aligned}
& \limsup _{n \rightarrow \infty}\left\langle(\mu F-\gamma V) x^{*}, x_{n}-x^{*}\right\rangle \\
& \quad=\lim _{i \rightarrow \infty}\left\langle(\mu F-\gamma V) x^{*}, x_{n_{i}}-x^{*}\right\rangle .
\end{aligned}
$$

Without loss of generality, we may further assume that $x_{n_{i}} \rightarrow$ $\tilde{x}$; then $\tilde{x} \in \Xi$ as we just proved. Since $x^{*}$ is a solution of the THVIP (8), we get

$$
\begin{array}{r}
\limsup _{n \rightarrow \infty}\left\langle(\mu F-\gamma V) x^{*}, x_{n}-x^{*}\right\rangle \\
=\left\langle(\mu F-\gamma V) x^{*}, \tilde{x}-x^{*}\right\rangle \geq 0 .
\end{array}
$$


From (11) and (41), it follows that (noticing that $x_{n+1}=P_{C} w_{n}$ and $0<\gamma \leq \tau$ )

$$
\begin{aligned}
\left\|x_{n+1}-x^{*}\right\|^{2}= & \left\langle w_{n}-x^{*}, x_{n+1}-x^{*}\right\rangle \\
& +\left\langle P_{C} w_{n}-w_{n}, P_{C} w_{n}-x^{*}\right\rangle \\
\leq & \left\langle w_{n}-x^{*}, x_{n+1}-x^{*}\right\rangle \\
= & \left\langle\left(I-\lambda_{n} \mu F\right) z_{n}-\left(I-\lambda_{n} \mu F\right) x^{*}, x_{n+1}-x^{*}\right\rangle \\
& +\delta_{n} \lambda_{n} \gamma\left\langle V x_{n}-V x^{*}, x_{n+1}-x^{*}\right\rangle \\
& +\lambda_{n}\left(1-\delta_{n}\right) \gamma\left\langle S x_{n}-S x^{*}, x_{n+1}-x^{*}\right\rangle \\
& +\delta_{n} \lambda_{n}\left\langle(\gamma V-\mu F) x^{*}, x_{n+1}-x^{*}\right\rangle \\
& +\lambda_{n}\left(1-\delta_{n}\right)\left\langle(\gamma S-\mu F) x^{*}, x_{n+1}-x^{*}\right\rangle \\
\leq & \left(1-\lambda_{n} \tau\right)\left\|z_{n}-x^{*}\right\|\left\|x_{n+1}-x^{*}\right\| \\
& +\left[\delta_{n} \lambda_{n} \gamma \rho+\lambda_{n}\left(1-\delta_{n}\right) \gamma\right] \\
& \times\left\|x_{n}-x^{*}\right\|\left\|x_{n+1}-x^{*}\right\| \\
& +\delta_{n} \lambda_{n}\left\langle(\gamma V-\mu F) x^{*}, x_{n+1}-x^{*}\right\rangle \\
& +\lambda_{n}\left(1-\delta_{n}\right)\left\langle(\gamma S-\mu F) x^{*}, x_{n+1}-x^{*}\right\rangle \\
& +\left(1-\lambda_{n} \tau\right) \frac{1}{2}\left(\left\|z_{n}-x^{*}\right\|^{2}+\left\|x_{n+1}-x^{*}\right\|^{2}\right) \\
& +\frac{1}{2}\left(\left\|x_{n}-x^{*}\right\|^{2}+\left\|x_{n+1}-x^{*}\right\|^{2}\right) \\
& +\left[\delta_{n} \lambda_{n} \gamma \rho+\lambda_{n}\left(1-\delta_{n}\right) \gamma\right] \\
& +\lambda_{n}\left(1-\delta_{n} \tau\right)\left\langle(\gamma S-\mu F) x^{*}, x_{n+1}-x^{*}\right\rangle \\
& \times \frac{1}{2}\left(\left\|x_{n}-x^{*}\right\|^{2}+\left\|x_{n+1}-x^{*}\right\|^{2}\right) \\
& +\delta_{n} \lambda_{n}\left\langle(\gamma V-\mu F) x^{*}, x_{n+1}-x^{*}\right\rangle \\
& +\lambda_{n}\left(1-\delta_{n}\right)\left\langle(\gamma S-\mu F) x^{*}, x_{n+1}-x^{*}\right\rangle \\
& \left(1-\lambda_{n} \tau\right) \frac{1}{2}\left[\left(\left\|x_{n}-x^{*}\right\|+\sqrt{2} v_{n} \alpha_{n}\left\|x^{*}\right\|\right)^{2}\right. \\
& +\left[\delta_{n} \lambda_{n} \gamma \rho+\lambda_{n}\left(1-\delta_{n}\right) \gamma\right] \\
& \left.+\left\|x_{n}-x^{*}\right\|^{2}+\left\|x_{n+1}-x^{*}\right\|^{2}\right) \\
& \\
& \\
& \\
& \\
&
\end{aligned}
$$

$$
\begin{aligned}
& +\delta_{n} \lambda_{n}\left\langle(\gamma V-\mu F) x^{*}, x_{n+1}-x^{*}\right\rangle \\
& +\lambda_{n}\left(1-\delta_{n}\right)\left\langle(\gamma S-\mu F) x^{*}, x_{n+1}-x^{*}\right\rangle \\
& \leq \\
& \left.+1-\delta_{n} \lambda_{n} \gamma(1-\rho)\right] \\
& +\frac{1}{2}\left(\left\|x_{n}-x^{*}\right\|^{2}+\left\|x_{n+1}-x^{*}\right\|^{2}\right) \\
& +\delta_{n} \lambda_{n}\left\langle(\gamma V-\mu F) x^{*}, x_{n+1}-x^{*}\right\rangle \\
& +\lambda_{n}\left(1-\delta_{n}\right)\left\langle(\gamma S-\mu F) x^{*}, x_{n+1}-x^{*}\right\rangle \\
& +\alpha_{n} M_{2},
\end{aligned}
$$

where $M_{2}=\sup _{n \geq 0}\left\{2 v_{n}\left\|x^{*}\right\|\left(\sqrt{2}\left\|x_{n}-x^{*}\right\|+v_{n} \alpha_{n}\left\|x^{*}\right\|\right)\right\}<\infty$. It turns out that

$$
\begin{aligned}
\left\|x_{n+1}-x^{*}\right\|^{2} \\
\leq \frac{1-\delta_{n} \lambda_{n} \gamma(1-\rho)}{1+\delta_{n} \lambda_{n} \gamma(1-\rho)}\left\|x_{n}-x^{*}\right\|^{2} \\
+\frac{2}{1+\delta_{n} \lambda_{n} \gamma(1-\rho)} \\
\times\left[\delta_{n} \lambda_{n}\left\langle(\gamma V-\mu F) x^{*}, x_{n+1}-x^{*}\right\rangle\right. \\
\quad+\lambda_{n}\left(1-\delta_{n}\right) \\
\left.\quad \times\left\langle(\gamma S-\mu F) x^{*}, x_{n+1}-x^{*}\right\rangle+\alpha_{n} M_{2}\right] \\
\leq\left[1-\delta_{n} \lambda_{n} \gamma(1-\rho)\right]\left\|x_{n}-x^{*}\right\|^{2} \\
+\frac{2}{1+\delta_{n} \lambda_{n} \gamma(1-\rho)} \\
\times\left[\delta_{n} \lambda_{n}\left\langle(\gamma V-\mu F) x^{*}, x_{n+1}-x^{*}\right\rangle\right. \\
\quad+\lambda_{n}\left(1-\delta_{n}\right) \\
\left.\quad\left\langle(\gamma S-\mu F) x^{*}, x_{n+1}-x^{*}\right\rangle\right]+2 \alpha_{n} M_{2} .
\end{aligned}
$$

However, from $x^{*} \in \Xi$ and condition (C6) we obtain that

$$
\begin{aligned}
\langle(\gamma S & \left.-\mu F) x^{*}, x_{n+1}-x^{*}\right\rangle \\
= & \left\langle(\gamma S-\mu F) x^{*}, x_{n+1}-P_{\mathrm{Fix}(T) \cap \Gamma} x_{n+1}\right\rangle \\
& +\left\langle(\gamma S-\mu F) x^{*}, P_{\mathrm{Fix}(T) \cap \Gamma} x_{n+1}-x^{*}\right\rangle \\
\leq & \left\langle(\gamma S-\mu F) x^{*}, x_{n+1}-P_{\mathrm{Fix}(T) \cap \Gamma} x_{n+1}\right\rangle \\
\leq & \left\|(\gamma S-\mu F) x^{*}\right\| d\left(x_{n+1}, \operatorname{Fix}(T) \cap \Gamma\right) \\
\leq & \left\|(\gamma S-\mu F) x^{*}\right\|\left(\frac{1}{\bar{k}}\left\|x_{n+1}-T x_{n+1}\right\|\right)^{1 / \theta} .
\end{aligned}
$$


On the other hand, from (41) we have

$$
\begin{aligned}
\left\|z_{n}-p\right\|^{2} \leq & \left(\left\|x_{n}-p\right\|+\sqrt{2} v_{n} \alpha_{n}\|p\|\right)^{2} \\
& +\left(1-\beta_{n}\right)\left(v_{n}^{2}\left(\alpha_{n}+L\right)^{2}-1\right)\left\|x_{n}-y_{n}\right\|^{2} \\
& -\frac{\beta_{n}}{1-\beta_{n}}\left\|z_{n}-x_{n}\right\|^{2} \\
\leq & \left(\left\|x_{n}-p\right\|+\sqrt{2} v_{n} \alpha_{n}\|p\|\right)^{2} \\
& +\left(1-\beta_{n}\right)\left(v_{n}^{2}\left(\alpha_{n}+L\right)^{2}-1\right)\left\|x_{n}-y_{n}\right\|^{2},
\end{aligned}
$$

which, together with $\left\|x_{n}-z_{n}\right\|+\alpha_{n}=\mathrm{o}\left(\lambda_{n}^{2}\right)$, implies that

$$
\begin{aligned}
& (1-d)\left(1-b^{2}\left(\alpha_{n}+L\right)^{2}\right) \frac{\left\|x_{n}-y_{n}\right\|^{2}}{\lambda_{n}^{2}} \\
& \leq\left(1-\beta_{n}\right)\left(1-v_{n}^{2}\left(\alpha_{n}+L\right)^{2}\right) \frac{\left\|x_{n}-y_{n}\right\|^{2}}{\lambda_{n}^{2}} \\
& \leq \frac{\left(\left\|x_{n}-p\right\|+\sqrt{2} v_{n} \alpha_{n}\|p\|\right)^{2}-\left\|z_{n}-p\right\|^{2}}{\lambda_{n}^{2}} \\
& \leq \frac{\left\|x_{n}-z_{n}\right\|+\sqrt{2} b \alpha_{n}\|p\|}{\lambda_{n}^{2}} \\
& \quad \times\left[\left\|x_{n}-p\right\|+\left\|z_{n}-p\right\|+\sqrt{2} b \alpha_{n}\|p\|\right] \longrightarrow 0
\end{aligned}
$$

That is, $\left\|x_{n}-y_{n}\right\|=o\left(\lambda_{n}\right)$. Observe that

$$
\begin{aligned}
z_{n}-x_{n} & =\gamma_{n}\left(t_{n}-x_{n}\right)+\sigma_{n}\left(T t_{n}-x_{n}\right) \\
& =\gamma_{n}\left(t_{n}-x_{n}\right)+\sigma_{n}\left(T t_{n}-T x_{n}\right)+\sigma_{n}\left(T x_{n}-x_{n}\right)
\end{aligned}
$$

Since $\left(\gamma_{n}+\sigma_{n}\right) \zeta \leq \gamma_{n}$, utilizing Lemma 10 we get

$$
\begin{aligned}
& \frac{\left\|\sigma_{n}\left(T x_{n}-x_{n}\right)\right\|}{\lambda_{n}} \\
& \quad=\frac{\left\|z_{n}-x_{n}-\left[\gamma_{n}\left(t_{n}-x_{n}\right)+\sigma_{n}\left(T t_{n}-T x_{n}\right)\right]\right\|}{\lambda_{n}} \\
& \leq \frac{\left\|z_{n}-x_{n}\right\|+\left\|\gamma_{n}\left(t_{n}-x_{n}\right)+\sigma_{n}\left(T t_{n}-T x_{n}\right)\right\|}{\lambda_{n}} \\
& \leq \frac{\left\|z_{n}-x_{n}\right\|+\left\|t_{n}-x_{n}\right\|}{\lambda_{n}} \\
& \leq \frac{\left\|z_{n}-x_{n}\right\|+\left\|t_{n}-y_{n}\right\|+\left\|y_{n}-x_{n}\right\|}{\lambda_{n}}
\end{aligned}
$$

$$
\begin{aligned}
= & \frac{\left\|z_{n}-x_{n}\right\|+\left\|P_{C}\left(x_{n}-v_{n} A_{n} y_{n}\right)-P_{C}\left(x_{n}-v_{n} A_{n} x_{n}\right)\right\|}{\lambda_{n}} \\
& +\frac{\left\|y_{n}-x_{n}\right\|}{\lambda_{n}} \\
\leq & \frac{\left\|z_{n}-x_{n}\right\|+\left\|v_{n} A_{n} y_{n}-v_{n} A_{n} x_{n}\right\|+\left\|y_{n}-x_{n}\right\|}{\lambda_{n}} \\
\leq & \frac{\left\|z_{n}-x_{n}\right\|+v_{n}\left(\alpha_{n}+L\right)\left\|y_{n}-x_{n}\right\|+\left\|y_{n}-x_{n}\right\|}{\lambda_{n}} \\
\leq & \frac{\left\|z_{n}-x_{n}\right\|+2\left\|y_{n}-x_{n}\right\|}{\lambda_{n}} \longrightarrow 0 \text { as } n \longrightarrow \infty .
\end{aligned}
$$

That is, $\left\|\sigma_{n}\left(T x_{n}-x_{n}\right)\right\|=o\left(\lambda_{n}\right)$. Taking into account $\lim \inf _{n \rightarrow \infty} \sigma_{n}>0$, we have $\left\|x_{n}-T x_{n}\right\|=o\left(\lambda_{n}\right)$. Furthermore, utilizing Lemma 7 (i), we have

$$
\begin{aligned}
&\left\|x_{n+1}-T x_{n+1}\right\| \\
& \leq\left\|x_{n+1}-T x_{n}\right\|+\left\|T x_{n}-T x_{n+1}\right\| \\
& \leq \frac{1+\zeta}{1-\zeta}\left\|x_{n}-x_{n+1}\right\| \\
&+\left\|\lambda_{n} \gamma\left(\delta_{n} V x_{n}+\left(1-\delta_{n}\right) S x_{n}\right)+\left(I-\lambda_{n} \mu F\right) z_{n}-T x_{n}\right\| \\
& \leq \frac{1+\zeta}{1-\zeta}\left\|x_{n}-x_{n+1}\right\| \\
&+\left\|z_{n}-T x_{n}\right\|+\lambda_{n}\left\|\gamma\left(\delta_{n} V x_{n}+\left(1-\delta_{n}\right) S x_{n}\right)-\mu F z_{n}\right\| \\
& \leq \frac{1+\zeta}{1-\zeta}\left\|x_{n}-x_{n+1}\right\| \\
&+\left\|z_{n}-x_{n}\right\|+\left\|x_{n}-T x_{n}\right\| \\
&+\lambda_{n}\left\|\gamma \delta_{n}\left(V x_{n}-S x_{n}\right)+\gamma S x_{n}-\mu F z_{n}\right\| \\
& \leq \frac{1+\zeta}{1-\zeta}\left\|x_{n}-x_{n+1}\right\| \\
&+\left\|z_{n}-x_{n}\right\|+\left\|x_{n}-T x_{n}\right\|+\lambda_{n} M M_{0},
\end{aligned}
$$

where $M_{0}=\sup _{n \geq 0}\left\|\gamma \delta_{n}\left(V x_{n}-S x_{n}\right)+\gamma S x_{n}-\mu F z_{n}\right\|<\infty$. Hence, for a big enough constant $\bar{k}_{1}>0$, from (77), we have

$$
\begin{aligned}
& \left\langle(\gamma S-\mu F) x^{*}, x_{n+1}-x^{*}\right\rangle \\
& \leq \bar{k}_{1}\left(\lambda_{n}+\left\|x_{n}-x_{n+1}\right\|\right. \\
& \left.\quad+\left\|z_{n}-x_{n}\right\|+\left\|x_{n}-T x_{n}\right\|\right)^{1 / \theta} \\
& \leq \bar{k}_{1} \lambda_{n}^{1 / \theta}\left(1+\frac{\left\|x_{n}-x_{n+1}\right\|+\left\|z_{n}-x_{n}\right\|+\left\|x_{n}-T x_{n}\right\|}{\lambda_{n}}\right)^{1 / \theta} .
\end{aligned}
$$


Combining (76)-(83), we get

$$
\begin{aligned}
\| x_{n+1} & -x^{*} \|^{2} \\
\leq & {\left[1-\delta_{n} \lambda_{n} \gamma(1-\rho)\right]\left\|x_{n}-x^{*}\right\|^{2} } \\
& +\frac{2}{1+\delta_{n} \lambda_{n} \gamma(1-\rho)} \\
& \times\left[\delta_{n} \lambda_{n}\left\langle(\gamma V-\mu F) x^{*}, x_{n+1}-x^{*}\right\rangle\right. \\
& \left.+\lambda_{n}\left(1-\delta_{n}\right)\left\langle(\gamma S-\mu F) x^{*}, x_{n+1}-x^{*}\right\rangle\right] \\
& +2 \alpha_{n} M_{2} \\
\leq & {\left[1-\delta_{n} \lambda_{n} \gamma(1-\rho)\right]\left\|x_{n}-x^{*}\right\|^{2} } \\
+ & \frac{2 \delta_{n} \lambda_{n}}{1+\delta_{n} \lambda_{n} \gamma(1-\rho)} \\
& \times\left[(\gamma V-\mu F) x^{*}, x_{n+1}-x^{*}\right\rangle \\
& +2 \alpha_{n} M_{2} \\
& +\frac{k_{1} \lambda_{n}^{1 / \theta}}{\delta_{n}} \\
& \left.\times\left(1-\frac{\left\|x_{n}-x_{n+1}\right\|+\left\|z_{n}-x_{n}\right\|+\left\|x_{n}-T x_{n}\right\|}{\lambda_{n}}\right)^{1 / \theta}\right]
\end{aligned}
$$

where $s_{n}=\delta_{n} \lambda_{n} \gamma(1-\rho)$ and

$$
\begin{aligned}
\mu_{n}= & \frac{2 \delta_{n} \lambda_{n}}{1+} \delta_{n} \lambda_{n} \gamma(1-\rho) \\
\times & {\left[\left\langle(\gamma V-\mu F) x^{*}, x_{n+1}-x^{*}\right\rangle\right.} \\
& +\frac{\bar{k}_{1} \lambda_{n}^{1 / \theta}}{\delta_{n}} \\
& \left.\quad \times\left(1+\frac{\left\|x_{n}-x_{n+1}\right\|+\left\|z_{n}-x_{n}\right\|+\left\|x_{n}-T x_{n}\right\|}{\lambda_{n}}\right)^{1 / \theta}\right] .
\end{aligned}
$$

Now condition (C5) implies that $\sum_{n=0}^{\infty} s_{n}=\infty$. Moreover, since $\left\|x_{n+1}-x_{n}\right\|+\left\|z_{n}-x_{n}\right\|+\left\|x_{n}-T x_{n}\right\|=o\left(\lambda_{n}\right)$, conditions (C7) and (74) imply that

$$
\limsup _{n \rightarrow \infty} \frac{\mu_{n}}{s_{n}} \leq 0 .
$$

Therefore, we can apply Lemma 8 to (84) to conclude that $x_{n} \rightarrow x^{*}$. The proof of part (a) is complete. It is easy to see that part (b) now becomes a straightforward consequence of part (a) since, if $V=0$, THVIP (8) reduces to the VIP in part (b). This completes the proof.
Next we consider a special case of Problem II. In Problem II, put $\mu=2, F=(1 / 2) I$ and $\gamma=\tau=1$. In this case, the objective is to find $x^{*} \in \Xi$ such that

$$
\left\langle(I-V) x^{*}, x-x^{*}\right\rangle \geq 0, \quad \forall x \in \Xi,
$$

where $\Xi$ denotes the solution set of the following hierarchical variational inequality problem (HVIP) of finding $z^{*} \epsilon$ $\operatorname{Fix}(T) \cap \Gamma$ such that

$$
\left\langle(I-S) z^{*}, z-z^{*}\right\rangle \geq 0, \quad \forall z \in \operatorname{Fix}(T) \cap \Gamma .
$$

Corollary 15. Let $A$ be a $1 / L$-inverse strongly monotone mapping, $V: C \rightarrow \mathscr{H}$ be a $\rho$-contraction with coefficient $\rho \in[0,1)$, $S: C \rightarrow C$ be a nonexpansive mapping, and $T: C \rightarrow C$ be a $\zeta$-strictly pseudocontractive mapping. Assume that the solution set $\Xi$ of the HVIP (88) is nonempty and that the following conditions hold for the sequences $\left\{\alpha_{n}\right\} \subset[0, \infty),\left\{v_{n}\right\} \quad \subset$ $(0,1 / L),\left\{\gamma_{n}\right\} \subset[0,1)$, and $\left\{\beta_{n}\right\},\left\{\delta_{n}\right\},\left\{\sigma_{n}\right\},\left\{\lambda_{n}\right\} \subset(0,1)$ :

(C1) $\sum_{n=0}^{\infty} \alpha_{n}<\infty$ and $\lim _{n \rightarrow \infty}\left(\alpha_{n} / \lambda_{n}^{2}\right)=0$;

(C2) $0<\lim \inf _{n \rightarrow \infty} v_{n} \leq \lim \sup _{n \rightarrow \infty} v_{n}<1 / L$;

(C3) $\beta_{n}+\gamma_{n}+\sigma_{n}=1$ and $\left(\gamma_{n}+\sigma_{n}\right) \zeta \leq \gamma_{n}$ for all $n \geq 0$;

(C4) $0<\lim \inf _{n \rightarrow \infty} \beta_{n} \leq \lim \sup _{n \rightarrow \infty} \beta_{n}<1$ and $\lim \inf _{n \rightarrow \infty} \sigma_{n}>0$;

(C5) $\lim _{n \rightarrow \infty} \lambda_{n}=0, \lim _{n \rightarrow \infty} \delta_{n}=0$ and $\sum_{n=0}^{\infty} \delta_{n} \lambda_{n}=\infty$;

(C6) there are constants $\bar{k}, \theta>0$ satisfying $\|x-T x\| \geq$ $\bar{k}[d(x, \operatorname{Fix}(T) \cap \Gamma)]^{\theta}$ for each $x \in C ;$

(C7) $\lim _{n \rightarrow \infty}\left(\lambda_{n}^{1 / \theta} / \delta_{n}\right)=0$.

One has

(a) If $\left\{x_{n}\right\}$ is the sequence generated by the iterative scheme

$$
\begin{gathered}
x_{0}=x \in C \text { chosen arbitrarily, } \\
y_{n}=P_{C}\left(x_{n}-v_{n} A_{n} x_{n}\right), \\
z_{n}=\beta_{n} x_{n}+\gamma_{n} P_{C}\left(x_{n}-v_{n} A_{n} y_{n}\right)+\sigma_{n} T P_{C}\left(x_{n}-v_{n} A_{n} y_{n}\right), \\
x_{n+1}=P_{C}\left[\lambda_{n}\left(\delta_{n} V x_{n}+\left(1-\delta_{n}\right) S x_{n}\right)\right. \\
\left.+\left(1-\lambda_{n}\right) z_{n}\right], \quad \forall n \geq 0
\end{gathered}
$$

and $\left\{S x_{n}\right\}$ is bounded, then $\left\{x_{n}\right\}$ converges strongly to the point $x^{*} \in \operatorname{Fix}(T) \cap \Gamma$ which is a unique solution of THVIP (87) provided that $\left\|x_{n+1}-x_{n}\right\|+\left\|x_{n}-z_{n}\right\|=$ $o\left(\lambda_{n}^{2}\right)$.

(b) If $\left\{x_{n}\right\}$ is the sequence generated by the iterative scheme

$$
\begin{gathered}
x_{0}=x \in C \text { chosen arbitrarily, } \\
y_{n}=P_{C}\left(x_{n}-v_{n} A_{n} x_{n}\right), \\
z_{n}=\beta_{n} x_{n}+\gamma_{n} P_{C}\left(x_{n}-v_{n} A_{n} y_{n}\right) \\
+\sigma_{n} T P_{C}\left(x_{n}-v_{n} A_{n} y_{n}\right),
\end{gathered}
$$$$
x_{n+1}=P_{C}\left[\lambda_{n}\left(1-\delta_{n}\right) S x_{n}+\left(1-\lambda_{n}\right) z_{n}\right], \quad \forall n \geq 0
$$ 
and $\left\{S x_{n}\right\}$ is bounded, then $\left\{x_{n}\right\}$ converges strongly to a unique solution $x^{*}$ of the following VIP provided that $\left\|x_{n+1}-x_{n}\right\|+$ $\left\|x_{n}-z_{n}\right\|=o\left(\lambda_{n}^{2}\right)$ :

$$
\text { find } x^{*} \in \Xi \text { such that }\left\langle x^{*}, x-x^{*}\right\rangle \geq 0, \quad \forall x \in \Xi \text {; }
$$

that is, $x^{*}$ is the minimum-norm solution of HVIP (88).

Furthermore, applying Theorem 14 to Problem I, we get the result as below.

Corollary 16. Let $F: C \rightarrow \mathscr{H}$ be a $\kappa$-Lipschitzian and $\eta$-strongly monotone operator with constants $\kappa, \eta>0$, respectively, $V: C \rightarrow \mathscr{H}$ be a $\rho$-contraction with coefficient $\rho \in[0,1), S: C \rightarrow C$ be a nonexpansive mapping, and $T_{i}:$ $C \rightarrow C$ be a $\zeta_{i}$-strictly pseudocontractive mapping for $i=1,2$. Let $0<\mu<2 \eta / \kappa^{2}$ and $0<\gamma \leq \tau$, where $\tau=1-$ $\sqrt{1-\mu\left(2 \eta-\mu \kappa^{2}\right)}$. Assume that the solution set $\Xi$ of the HVIP in Problem I is nonempty and that the following conditions hold for the sequences $\left\{v_{n}\right\} \subset\left(0,\left(1-\zeta_{2}\right) / 2\right),\left\{\gamma_{n}\right\} \subset[0,1)$ and $\left\{\beta_{n}\right\},\left\{\delta_{n}\right\},\left\{\sigma_{n}\right\},\left\{\lambda_{n}\right\} \subset(0,1)$ :

(C1) $0<\lim \inf _{n \rightarrow \infty} \nu_{n} \leq \lim \sup _{n \rightarrow \infty} \nu_{n}<\left(1-\zeta_{2}\right) / 2$;

(C2) $\beta_{n}+\gamma_{n}+\sigma_{n}=1$ and $\left(\gamma_{n}+\sigma_{n}\right) \zeta \leq \gamma_{n}$ for all $n \geq 0$;

(C3) $0<\lim \inf _{n \rightarrow \infty} \beta_{n} \leq \lim \sup _{n \rightarrow \infty} \beta_{n}<1$ and $\liminf _{n \rightarrow \infty} \sigma_{n}>0$;

(C4) $\lim _{n \rightarrow \infty} \lambda_{n}=0, \lim _{n \rightarrow \infty} \delta_{n}=0$ and $\sum_{n=0}^{\infty} \delta_{n} \lambda_{n}=\infty$;

(C5) there are constants $\bar{k}, \theta>0$ satisfying $\left\|x-T_{1} x\right\| \geq$ $\bar{k}\left[d\left(x, \operatorname{Fix}\left(T_{1}\right) \cap \operatorname{Fix}\left(T_{2}\right)\right)\right]^{\theta}$ for each $x \in C ;$

(C6) $\lim _{n \rightarrow \infty}\left(\lambda_{n}^{1 / \theta} / \delta_{n}\right)=0$.

One has the following.

(a) If $\left\{x_{n}\right\}$ is the sequence generated by

$$
\begin{gathered}
x_{0}=x \in C \text { chosen arbitrarily, } \\
y_{n}=x_{n}-v_{n}\left(I-T_{2}\right) x_{n}, \\
z_{n}=\beta_{n} x_{n}+\gamma_{n} P_{C}\left(x_{n}-v_{n}\left(I-T_{2}\right) y_{n}\right) \\
+\sigma_{n} T P_{C}\left(x_{n}-v_{n}\left(I-T_{2}\right) y_{n}\right), \\
x_{n+1}=P_{C}\left[\lambda_{n} \gamma\left(\delta_{n} V x_{n}+\left(1-\delta_{n}\right) S x_{n}\right)\right. \\
\left.+\left(I-\lambda_{n} \mu F\right) z_{n}\right], \quad \forall n \geq 0,
\end{gathered}
$$

such that $\left\{S x_{n}\right\}$ is bounded, then $\left\{x_{n}\right\}$ converges strongly to the point $x^{*} \in \operatorname{Fix}\left(T_{1}\right) \cap \operatorname{Fix}\left(T_{2}\right)$ which is a unique solution of Problem I provided that $\left\|x_{n+1}-x_{n}\right\|+\| x_{n}-$ $z_{n} \|=o\left(\lambda_{n}^{2}\right)$. (b) If $\left\{x_{n}\right\}$ is the sequence generated by

$$
\begin{gathered}
x_{0}=x \in C \text { chosen arbitrarily, } \\
y_{n}=x_{n}-v_{n}\left(I-T_{2}\right) x_{n}, \\
z_{n}=\beta_{n} x_{n}+\gamma_{n} P_{C}\left(x_{n}-v_{n}\left(I-T_{2}\right) y_{n}\right) \\
+\sigma_{n} T P_{C}\left(x_{n}-v_{n}\left(I-T_{2}\right) y_{n}\right), \\
x_{n+1}=P_{C}\left(\lambda_{n}\left(1-\delta_{n}\right) \gamma S x_{n}\right. \\
\left.\quad+\left(I-\lambda_{n} \mu F\right) z_{n}\right], \quad \forall n \geq 0,
\end{gathered}
$$

such that $\left\{S x_{n}\right\}$ is bounded, then $\left\{x_{n}\right\}$ converges strongly to a unique solution $x^{*}$ of the following VIP provided that $\| x_{n+1}-$ $x_{n}\|+\| x_{n}-z_{n} \|=o\left(\lambda_{n}^{2}\right)$ :

find $x^{*} \in \Xi$ such that $\left\langle F x^{*}, x-x^{*}\right\rangle \geq 0, \quad \forall x \in \Xi$.

Proof. In Theorem 14, we put $T=T_{1}$ and $A=I-T_{2}$ where $T_{i}: C \rightarrow C$ is $\zeta_{i}$-strictly pseudocontractive for $i=1,2$. Taking $L=2 /\left(1-\zeta_{2}\right)$ and $\alpha_{n}=0$ for all $n \geq 0$, we know that $A: C \rightarrow \mathscr{H}$ is a $1 / L$-inverse strongly monotone mapping such that $\Gamma=\operatorname{Fix}\left(T_{2}\right)$. In the scheme (11), we have

$$
\begin{aligned}
y_{n} & =P_{C}\left(x_{n}-v_{n} A_{n} x_{n}\right)=P_{C}\left(\left(1-v_{n}\right) x_{n}+v_{n} T_{2} x_{n}\right) \\
& =x_{n}-v_{n}\left(I-T_{2}\right) x_{n} .
\end{aligned}
$$

Utilizing Theorem 14, we obtain desired result.

On the other hand, we also derive the following strong convergence result of Algorithm I for finding a unique solution of Problem III.

Theorem 17. Let $F: C \rightarrow \mathscr{H}$ be a $\kappa$-Lipschitzian and $\eta$-strongly monotone operator with constants $\kappa, \eta>0$, respectively, $A$ be a $1 / L$-inverse strongly monotone mapping, $V: C \rightarrow \mathscr{H}$ be a $\rho$-contraction with coefficient $\rho \in[0,1)$, $S: C \rightarrow C$ be a nonexpansive mapping, and $T: C \rightarrow C$ be a $\zeta$-strictly pseudocontractive mapping. Let $0<\mu<2 \eta / \kappa^{2}$ and $0<\gamma \leq \tau$, where $\tau=1-\sqrt{1-\mu\left(2 \eta-\mu \kappa^{2}\right)}$. Assume that Problem III has a solution and that the following conditions hold for the sequences $\left\{\alpha_{n}\right\} \subset(0, \infty),\left\{\nu_{n}\right\} \subset(0,1 / L),\left\{\gamma_{n}\right\} \subset$ $[0,1)$ and $\left\{\beta_{n}\right\},\left\{\delta_{n}\right\},\left\{\sigma_{n}\right\},\left\{\lambda_{n}\right\} \subset(0,1)$ :

(C1) $\sum_{n=0}^{\infty} \alpha_{n}<\infty$;

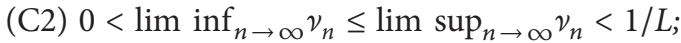

(C3) $\beta_{n}+\gamma_{n}+\sigma_{n}=1$ and $\left(\gamma_{n}+\sigma_{n}\right) \zeta \leq \gamma_{n}$ for all $n \geq 0$;

(C4) $0<\lim \inf _{n \rightarrow \infty} \beta_{n} \leq \lim \sup _{n \rightarrow \infty} \beta_{n}<1$ and $\lim \inf _{n \rightarrow \infty} \sigma_{n}>0$

(C5) $0<\lim \inf _{n \rightarrow \infty} \delta_{n} \leq \lim \sup _{n \rightarrow \infty} \delta_{n}<1$;

(C6) $\lim _{n \rightarrow \infty} \lambda_{n}=0$ and $\sum_{n=0}^{\infty} \lambda_{n}=\infty$.

One has the following.

(a) If $\left\{x_{n}\right\}$ is the sequence generated by the scheme (11) and $\left\{S x_{n}\right\}$ is bounded, then $\left\{x_{n}\right\}$ converges strongly to a unique solution of Problem III provided that $\lim _{n \rightarrow \infty}\left\|x_{n+1}-x_{n}\right\|=0$. 
(b) If $\left\{x_{n}\right\}$ is the sequence generated by the scheme (12) and $\left\{S x_{n}\right\}$ is bounded, then $\left\{x_{n}\right\}$ converges strongly to a unique solution $x^{*} \in \operatorname{Fix}(T) \cap \Gamma$ of the following system of variational inequalities provided that $\lim _{n \rightarrow \infty}\left\|x_{n+1}-x_{n}\right\|=0$ :

$$
\begin{gathered}
\left\langle F x^{*}, x-x^{*}\right\rangle \geq 0, \quad \forall x \in \operatorname{Fix}(T) \cap \Gamma, \\
\left\langle(\mu F-\gamma S) x^{*}, y-x^{*}\right\rangle \geq 0, \quad \forall y \in \operatorname{Fix}(T) \cap \Gamma .
\end{gathered}
$$

Proof. We treat only case (a); that is, the sequence $\left\{x_{n}\right\}$ is generated by scheme (11). First of all, it is seen easily that $0<\gamma \leq \tau$ and $\kappa \geq \eta \Leftrightarrow \mu \eta \geq \tau$. Hence it follows from the $\rho$-contractiveness of $V$ and $\gamma \rho<\gamma \leq \tau \leq \mu \eta$ that $\mu F-\gamma V$ is $(\mu \eta-\gamma \rho)$-strongly monotone and Lipschitz continuous. So, there exists a unique solution $x^{*}$ of the following VIP:

find $x^{*} \in \operatorname{Fix}(T) \cap \Gamma$ such that $\left\langle(\mu F-\gamma V) x^{*}, x-x^{*}\right\rangle \geq 0$,

$$
\forall x \in \operatorname{Fix}(T) \cap \Gamma \text {. }
$$

Consequently, it is easy to see that Problem III has a unique solution $x^{*} \in \operatorname{Fix}(T) \cap \Gamma$. In addition, taking into account condition (C5), without loss of generality we may assume that $\left\{\delta_{n}\right\} \subset[a, b]$ for some $a, b \in(0,1)$.

Next we divide the rest of the proof into several steps.

Step 1 ( $\left\{x_{n}\right\}$ is bounded). Indeed, repeating the same argument as in Step 1 of the proof of Theorem 14 we can derive the claim.

Step $2\left(\lim _{n \rightarrow \infty}\left\|x_{n}-y_{n}\right\|=\lim _{n \rightarrow \infty}\left\|x_{n}-t_{n}\right\|=\lim _{n \rightarrow \infty} \| t_{n}-\right.$ $T t_{n} \|=0$ ). Indeed, repeating the same argument as in Step 2 of the proof of Theorem 14 we can derive the claim.

Step $3\left(\omega_{w}\left(x_{n}\right) \subset \operatorname{Fix}(T) \cap \Gamma\right)$. Indeed, repeating the same argument as in Step 3 of the proof of Theorem 14 we can derive the claim.

Step 4 ( $\left\{x_{n}\right\}$ converges strongly to a unique solution $x^{*}$ of Problem III). Indeed, according to $\left\|x_{n+1}-x_{n}\right\| \rightarrow 0$, we can take a subsequence $\left\{x_{n_{i}}\right\}$ of $\left\{x_{n}\right\}$ satisfying

$$
\begin{aligned}
& \limsup _{n \rightarrow \infty}\left\langle(\gamma V-\mu F) x^{*}, x_{n+1}-x^{*}\right\rangle \\
& \quad=\limsup _{n \rightarrow \infty}\left\langle(\gamma V-\mu F) x^{*}, x_{n}-x^{*}\right\rangle \\
& =\lim _{i \rightarrow \infty}\left\langle(\gamma V-\mu F) x^{*}, x_{n_{i}}-x^{*}\right\rangle .
\end{aligned}
$$

Without loss of generality, we may further assume that $x_{n_{i}} \rightarrow$ $\tilde{x}$; then $\tilde{x} \in \operatorname{Fix}(T) \cap \Gamma$ due to Step 3. Since $x^{*}$ is a solution of Problem III, we get

$$
\begin{gathered}
\limsup _{n \rightarrow \infty}\left\langle(\gamma V-\mu F) x^{*}, x_{n+1}-x^{*}\right\rangle \\
=\left\langle(\gamma V-\mu F) x^{*}, \tilde{x}-x^{*}\right\rangle \leq 0 .
\end{gathered}
$$

Repeating the same argument as that of (99), we have

$$
\limsup _{n \rightarrow \infty}\left\langle(\gamma S-\mu F) x^{*}, x_{n+1}-x^{*}\right\rangle \leq 0
$$

Repeating the same argument as that of (76) in the proof of Theorem 14, we obtain

$$
\begin{aligned}
\| x_{n+1}- & x^{*} \|^{2} \\
\leq & {\left[1-\delta_{n} \lambda_{n} \gamma(1-\rho)\right]\left\|x_{n}-x^{*}\right\|^{2} } \\
+ & \frac{2}{1+\delta_{n} \lambda_{n} \gamma(1-\rho)} \\
\times & {\left[\delta_{n} \lambda_{n}\left\langle(\gamma V-\mu F) x^{*}, x_{n+1}-x^{*}\right\rangle\right.} \\
& \left.+\lambda_{n}\left(1-\delta_{n}\right)\left\langle(\gamma S-\mu F) x^{*}, x_{n+1}-x^{*}\right\rangle\right] \\
+ & 2 \alpha_{n} M_{2} . \\
\text { Put } r_{n}= & 2 \alpha_{n} M_{2}, s_{n}=\delta_{n} \lambda_{n} \gamma(1-\rho) \text { and } \\
b_{n}= & \frac{2}{\gamma(1-\rho)\left[1+\delta_{n} \lambda_{n} \gamma(1-\rho)\right]} \\
& \times\left\langle(\gamma V-\mu F) x^{*}, x_{n+1}-x^{*}\right\rangle \\
& \quad+\frac{2\left(1-\delta_{n}\right)}{\delta_{n} \gamma(1-\rho)\left[1+\delta_{n} \lambda_{n} \gamma(1-\rho)\right]} \\
& \times\left\langle(\gamma S-\mu F) x^{*}, x_{n+1}-x^{*}\right\rangle .
\end{aligned}
$$

Then (101) is rewritten as

$$
\left\|x_{n+1}-x^{*}\right\|^{2} \leq\left(1-s_{n}\right)\left\|x_{n}-x^{*}\right\|^{2}+s_{n} b_{n}+r_{n} .
$$

In terms of conditions (C5) and (C6), we conclude from $0<$ $1-\rho \leq 1$ that

$$
\left\{s_{n}\right\} \subset(0,1], \quad \sum_{n=0}^{\infty} s_{n}=\infty .
$$

Note that

$$
\begin{gathered}
\frac{2}{\gamma(1-\rho)\left[1+\delta_{n} \lambda_{n} \gamma(1-\rho)\right]} \leq \frac{2}{\gamma(1-\rho)}, \\
\frac{2\left(1-\delta_{n}\right)}{\delta_{n} \gamma(1-\rho)\left[1+\delta_{n} \lambda_{n} \gamma(1-\rho)\right]} \leq \frac{2}{a \gamma(1-\rho)} .
\end{gathered}
$$

Consequently, utilizing Lemma 13 we obtain that

$$
\begin{aligned}
& \limsup _{n \rightarrow \infty} b_{n} \leq \limsup _{n \rightarrow \infty} \frac{2}{\gamma(1-\rho)\left[1+\delta_{n} \lambda_{n} \gamma(1-\rho)\right]} \\
& \times\left\langle(\gamma V-\mu F) x^{*}, x_{n+1}-x^{*}\right\rangle \\
&+\limsup _{n \rightarrow \infty} \frac{2\left(1-\delta_{n}\right)}{\delta_{n} \gamma(1-\rho)\left[1+\delta_{n} \lambda_{n} \gamma(1-\rho)\right]} \\
& \quad \times\left\langle(\gamma S-\mu F) x^{*}, x_{n+1}-x^{*}\right\rangle \\
& \leq 0 .
\end{aligned}
$$

So, this together with Lemma 8 leads to $\lim _{n \rightarrow \infty}\left\|x_{n}-x^{*}\right\|=0$. The proof is complete. 
Utilizing Theorem 17 we immediately derive the following result.

Corollary 18. Let $F: C \rightarrow \mathscr{H}$ be a $\kappa$-Lipschitzian and $\eta$-strongly monotone operator with constants $\kappa, \eta>0$, respectively, $A$ be a $1 / L$-inverse strongly monotone mapping, $V: C \rightarrow \mathscr{H}$ be a $\rho$-contraction with coefficient $\rho \in[0,1)$, and $T: C \rightarrow C$ be a $\zeta$-strictly pseudocontractive mapping such that $\operatorname{Fix}(T) \cap \Gamma \neq \emptyset$. Let $0<\mu<2 \eta / \kappa^{2}$ and $0<\gamma \leq \tau$, where $\tau=1-\sqrt{1-\mu\left(2 \eta-\mu \kappa^{2}\right)}$. Assume that the following conditions hold for the sequences $\left\{\alpha_{n}\right\} \subset(0, \infty),\left\{\nu_{n}\right\} \subset(0,1 / L),\left\{\gamma_{n}\right\} \subset$ $[0,1)$ and $\left\{\beta_{n}\right\},\left\{\sigma_{n}\right\},\left\{\lambda_{n}\right\} \subset(0,1)$ :

(C1) $\sum_{n=0}^{\infty} \alpha_{n}<\infty$;

(C2) $0<\lim \inf _{n \rightarrow \infty} v_{n} \leq \lim \sup _{n \rightarrow \infty} v_{n}<1 / L$;

(C3) $\beta_{n}+\gamma_{n}+\sigma_{n}=1$ and $\left(\gamma_{n}+\sigma_{n}\right) \zeta \leq \gamma_{n}$ for all $n \geq 0$;

(C4) $0<\lim \inf _{n \rightarrow \infty} \beta_{n} \leq \lim \sup _{n \rightarrow \infty} \beta_{n}<1$ and $\lim \inf _{n \rightarrow \infty} \sigma_{n}>0$;

(C5) $\lim _{n \rightarrow \infty} \lambda_{n}=0$ and $\sum_{n=0}^{\infty} \lambda_{n}=\infty$.

One has the following.

(a) If $\left\{x_{n}\right\}$ is the sequence generated by the scheme (13) and $\left\{V x_{n}\right\}$ is bounded, then $\left\{x_{n}\right\}$ converges strongly to a unique solution of the following VIP provided that $\lim _{n \rightarrow \infty}\left\|x_{n+1}-x_{n}\right\|=0$ :

find $x^{*} \in \operatorname{Fix}(T) \cap \Gamma$ such that $\left\langle(\mu F-\gamma V) x^{*}, x-x^{*}\right\rangle \geq 0$,

$\forall x \in \operatorname{Fix}(T) \cap \Gamma$.

(b) If $\left\{x_{n}\right\}$ is the sequence generated by the scheme (14), then $\left\{x_{n}\right\}$ converges strongly to a unique solution of the following VIP provided that $\lim _{n \rightarrow \infty}\left\|x_{n+1}-x_{n}\right\|=0$ :

find $x^{*} \in \operatorname{Fix}(T) \cap \Gamma$ such that $\left\langle F x^{*}, x-x^{*}\right\rangle \geq 0$,

$\forall x \in \operatorname{Fix}(T) \cap \Gamma$.

Proof. In Theorem 17, putting $S=V$, we know that the iterative scheme (11) reduces to (13) since there holds for any $\left\{\delta_{n}\right\} \subset(0,1)$

$$
\begin{aligned}
x_{n+1} & =P_{C}\left[\lambda_{n} \gamma\left(\delta_{n} V x_{n}+\left(1-\delta_{n}\right) S x_{n}\right)+\left(I-\lambda_{n} \mu F\right) z_{n}\right] \\
& =P_{C}\left[\lambda_{n} \gamma\left(\delta_{n} V x_{n}+\left(1-\delta_{n}\right) V x_{n}\right)+\left(I-\lambda_{n} \mu F\right) z_{n}\right] \\
& =P_{C}\left[\lambda_{n} \gamma V x_{n}+\left(I-\lambda_{n} \mu F\right) z_{n}\right] .
\end{aligned}
$$

In this case, the SVI (15) with VIP constraint is equivalent to the VIP (107). Thus, utilizing Theorem 17 (a) we obtain the desired conclusion (a). As for the conclusion (b), we immediately derive it from $S=V \equiv 0$ and Theorem 17 (b).

In addition, applying Theorem 17 to Problem IV, we derive the result as below.
Corollary 19. Let $F: C \rightarrow \mathscr{H}$ be a $\kappa$-Lipschitzian and $\eta$-strongly monotone operator with constants $\kappa, \eta>0$, respectively, $V: C \rightarrow \mathscr{H}$ be a $\rho$-contraction with coefficient $\rho \in[0,1), S: C \rightarrow C$ be a nonexpansive mapping, and $T_{i}: C \rightarrow C$ be a $\zeta_{i}$-strictly pseudocontractive mapping for $i=1$, 2. Let $0<\mu<2 \eta / \kappa^{2}$ and $0<\gamma \leq \tau$, where $\tau=$ $1-\sqrt{1-\mu\left(2 \eta-\mu \kappa^{2}\right)}$. Assume that Problem IV has a solution and that the following conditions hold for the sequences $\left\{\nu_{n}\right\} \subset$ $\left(0,\left(1-\zeta_{2}\right) / 2\right),\left\{\gamma_{n}\right\} \subset[0,1)$ and $\left\{\beta_{n}\right\},\left\{\delta_{n}\right\},\left\{\sigma_{n}\right\},\left\{\lambda_{n}\right\} \subset(0,1)$ :

(C1) $0<\liminf _{n \rightarrow \infty} v_{n} \leq \lim \sup _{n \rightarrow \infty} v_{n}<\left(1-\zeta_{2}\right) / 2$;

(C2) $\beta_{n}+\gamma_{n}+\sigma_{n}=1$ and $\left(\gamma_{n}+\sigma_{n}\right) \zeta \leq \gamma_{n}$ for all $n \geq 0$;

(C3) $0<\lim \inf _{n \rightarrow \infty} \beta_{n} \leq \lim \sup _{n \rightarrow \infty} \beta_{n}<1$ and $\lim _{\inf _{n \rightarrow \infty} \sigma_{n}>0 ;}$

(C4) $0<\lim \inf _{n \rightarrow \infty} \delta_{n} \leq \lim \sup _{n \rightarrow \infty} \delta_{n}<1$;

(C5) $\lim _{n \rightarrow \infty} \lambda_{n}=0$ and $\sum_{n=0}^{\infty} \lambda_{n}=\infty$.

One has the following.

(a) If $\left\{x_{n}\right\}$ is the sequence generated by the scheme in Corollary 16 (a) such that $\left\{S x_{n}\right\}$ is bounded, then $\left\{x_{n}\right\}$ converges strongly to a unique solution of Problem IV provided that $\lim _{n \rightarrow \infty}\left\|x_{n+1}-x_{n}\right\|=0$.

(b) If $\left\{x_{n}\right\}$ is the sequence generated by the scheme in Corollary $16(b)$ such that $\left\{S x_{n}\right\}$ is bounded, then $\left\{x_{n}\right\}$ converges strongly to a unique solution $x^{*} \in$ $\operatorname{Fix}\left(T_{1}\right) \cap \operatorname{Fix}\left(T_{2}\right)$ of the following system of variational inequalities provided that $\lim _{n \rightarrow \infty}\left\|x_{n+1}-x_{n}\right\|=0$ :

$$
\begin{gathered}
\left\langle F x^{*}, x-x^{*}\right\rangle \geq 0, \quad \forall x \in \operatorname{Fix}\left(T_{1}\right) \cap \operatorname{Fix}\left(T_{2}\right), \\
\left\langle(\mu F-\gamma S) x^{*}, y-x^{*}\right\rangle \geq 0, \quad \forall y \in \operatorname{Fix}\left(T_{1}\right) \cap \operatorname{Fix}\left(T_{2}\right) .
\end{gathered}
$$

\section{Acknowledgments}

In this research, first two authors were partially supported by the National Science Foundation of China (11071169), Innovation Program of Shanghai Municipal Education Commission (09ZZ133), and Leading Academic Discipline Project of Shanghai Normal University (DZL707). The third author was partially supported by a research project no. SR/S4/MS: 719/11 of Department of Science and Technology, Govternment of India. The fourth author was supported in part by the National Science Council of the Republic of China.

\section{References}

[1] P. E. Maingé and A. Moudafi, "Strong convergence of an iterative method for hierarchical fixed-point problems," Pacific Journal of Optimization, vol. 3, no. 3, pp. 529-538, 2007.

[2] A. Moudafi and P. E. Maingé, "Towards viscosity approximations of hierarchical fixed-point problems," Fixed Point Theory and Applications, vol. 2006, Article ID 95453, 10 pages, 2006.

[3] A. Cabot, "Proximal point algorithm controlled by a slowly vanishing term: applicationsto hierarchical minimization," SIAM Journal on Optimization, vol. 15, no. 2, pp. 555-572, 2005. 
[4] F. Cianciaruso, V. Colao, L. Muglia, and H. K. Xu, "On an implicit hierarchical fixed pointapproach to variational inequalities," Bulletin of the Australian Mathematical Society, vol. 80, no. 1, pp. 117-124, 2009.

[5] H. Iiduka, "A new iterative algorithm for the variational inequality problem over the fixedpoint set of a firmly nonexpansive mapping," Optimization, vol. 59, no. 6, pp. 873-885, 2010.

[6] H. Iiduka and I. Yamada, "A use of conjugate gradient direction for the convex optimization problem over the fixed point set of a nonexpansive mapping," SIAM Journal on Optimization, vol. 19, no. 4, pp. 1881-1893, 2008.

[7] G. Marino and H. K. Xu, "Explicit hierarchical fixed point approach to variational inequalities," Journal of Optimization Theory and Applications, vol. 149, no. 1, pp. 61-78, 2011.

[8] A. Moudafi, "Krasnoselski-Mann iteration for hierarchical fixed-point problems," Inverse Problems, vol. 23, no. 4, pp. 1635$1640,2007$.

[9] H. K. Xu, "Viscosity method for hierarchical fixed point approach to variational inequalities," Taiwanese Journal of Mathematics, vol. 14, no. 2, pp. 463-478, 2010.

[10] H. K. Xu, "An iterative approach to quadratic optimization," Journal of Optimization Theory and Applications, vol. 116, no. 3, pp. 659-678, 2003.

[11] P. L. Combettes, "A block-iterative surrogate constraint splitting method for quadratic signal recovery," IEEE Transactions on Signal Processing, vol. 51, no. 7, pp. 1771-1782, 2003.

[12] K. Slavakis and I. Yamada, "Robust wideband beamforming by the hybrid steepest descent method," IEEE Transactions on Signal Processing, vol. 55, no. 9, pp. 4511-4522, 2007.

[13] H. Iiduka, "Fixed point optimization algorithm and its application to power control in CDMA data networks," Mathematical Programming, vol. 133, no. 1-2, pp. 227-242, 2012.

[14] I. Yamada and N. Ogura, "Hybrid steepest descent method for variational inequality problem over the fixed point set of certain quasi-nonexpansive mappings," Numerical Functional Analysis and Optimization, vol. 25, no. 7-8, pp. 619-655, 2004.

[15] Y. Yao and Y. C. Liou, "Weak and strong convergence of Krasnoselski-Mann iteration for hierarchical fixed point problems," Inverse Problems, vol. 24, no. 1, Article ID 015015, 8 pages, 2008.

[16] Y. Yao, R. Chen, and H. K. Xu, "Schemes for finding minimumnorm solutions of variational inequalities," Nonlinear Analysis: Theory, Methods \& Applications, vol. 72, no. 7-8, pp. 3447-3456, 2010.

[17] L. C. Ceng, Y. C. Lin, and A. Petruşel, "Hybrid method for designing explicit hierarchical fixed point approach to monotone variational inequalities," Taiwanese Journal of Mathematics, vol. 16, no. 4, pp. 1531-1555, 2012.

[18] L. C. Ceng, Q. H. Ansari, M. M. Wong, and J. C. Yao, "Mann type hybrid extragradientmethod for variational inequalities, variational inclusions and fixed point problems," Fixed Point Theory, vol. 13, no. 2, pp. 403-422, 2012.

[19] A. Moudafi, "Viscosity approximation methods for fixed-points problems," Journal of Mathematical Analysis and Applications, vol. 241, no. 1, pp. 46-55, 2000.

[20] H. K. Xu, "Viscosity approximation methods for nonexpansive mappings," Journal of Mathematical Analysis and Applications, vol. 298, no. 1, pp. 279-291, 2004.

[21] G. Marino and H. K. Xu, "Weak and strong convergence theorems for strict pseudo-contractions in Hilbert spaces," Journal of Mathematical Analysis and Applications, vol. 329, no. 1, pp. 336-346, 2007.
[22] L. C. Ceng, Q. H. Ansari, and J. C. Yao, "Iterative methods for triple hierarchical variational inequalities in Hilbert spaces," Journal of Optimization Theory and Applications, vol. 151, no. 3, pp. 489-512, 2011.

[23] G. M. Korpelevič, "An extragradient method for finding saddle points and for other problems," Ėkonomika i Matematicheskie Metody, vol. 12, no. 4, pp. 747-756, 1976.

[24] D. Han and H. K. Lo, "Solving non-additive traffic assignment problems: a descent method for co-coercive variational inequalities," European Journal of Operational Research, vol. 159, no. 3, pp. 529-544, 2004.

[25] D. P. Bertsekas and E. M. Gafni, "Projection methods for variational inequalities with application to the traffic assignment problem," Mathematical Programming Study, no. 17, pp. 139-159, 1982.

[26] C. Byrne, "A unified treatment of some iterative algorithms in signal processing and image reconstruction," Inverse Problems, vol. 20, no. 1, pp. 103-120, 2004.

[27] P. L. Combettes, "Solving monotone inclusions via compositions of nonexpansive averaged operators," Optimization, vol. 53, no. 5-6, pp. 475-504, 2004.

[28] H. K. Xu, "Iterative algorithms for nonlinear operators," Journal of the London Mathematical Society. Second Series, vol. 66, no. 1, pp. 240-256, 2002.

[29] Y. Yao, Y. C. Liou, and S. M. Kang, "Approach to common elements of variational inequality problems and fixed point problems via a relaxed extragradient method," Computers \& Mathematics with Applications, vol. 59, no. 11, pp. 3472-3480, 2010.

[30] H. K. Xu and T. H. Kim, "Convergence of hybrid steepestdescent methods for variational inequalities," Journal of Optimization Theory and Applications, vol. 119, no. 1, pp. 185-201, 2003.

[31] J. Reinermann, "Über Fixpunkte kontrahierender Abbildungen und schwach konvergente Toeplitz-Verfahren," Archiv der Mathematik, vol. 20, pp. 59-64, 1969.

[32] L. C. Ceng, A. Petruşel, and J. C. Yao, "Strong convergence theorems of averaging iterations of nonexpansive nonselfmappings in Banach spaces," Fixed Point Theory, vol. 8, no. 2, pp. 219-236, 2007.

[33] R. T. Rockafellar, "On the maximality of sums of nonlinear monotone operators," Transactions of the American Mathematical Society, vol. 149, pp. 75-88, 1970.

[34] L. C. Ceng, A. Petruşel, and J. C. Yao, "Relaxed extragradient methods with regularization for general system of variational inequalities with constraints of split feasibility and fixed point problems," Abstract and Applied Analysis. In press. 


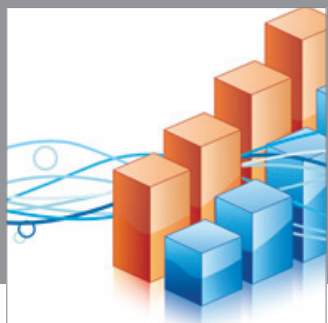

Advances in

Operations Research

mansans

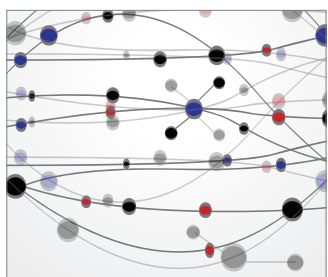

The Scientific World Journal
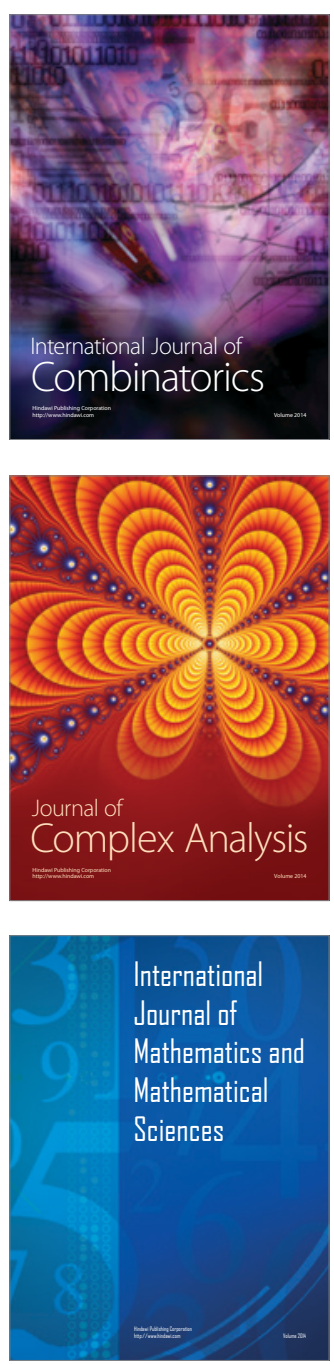
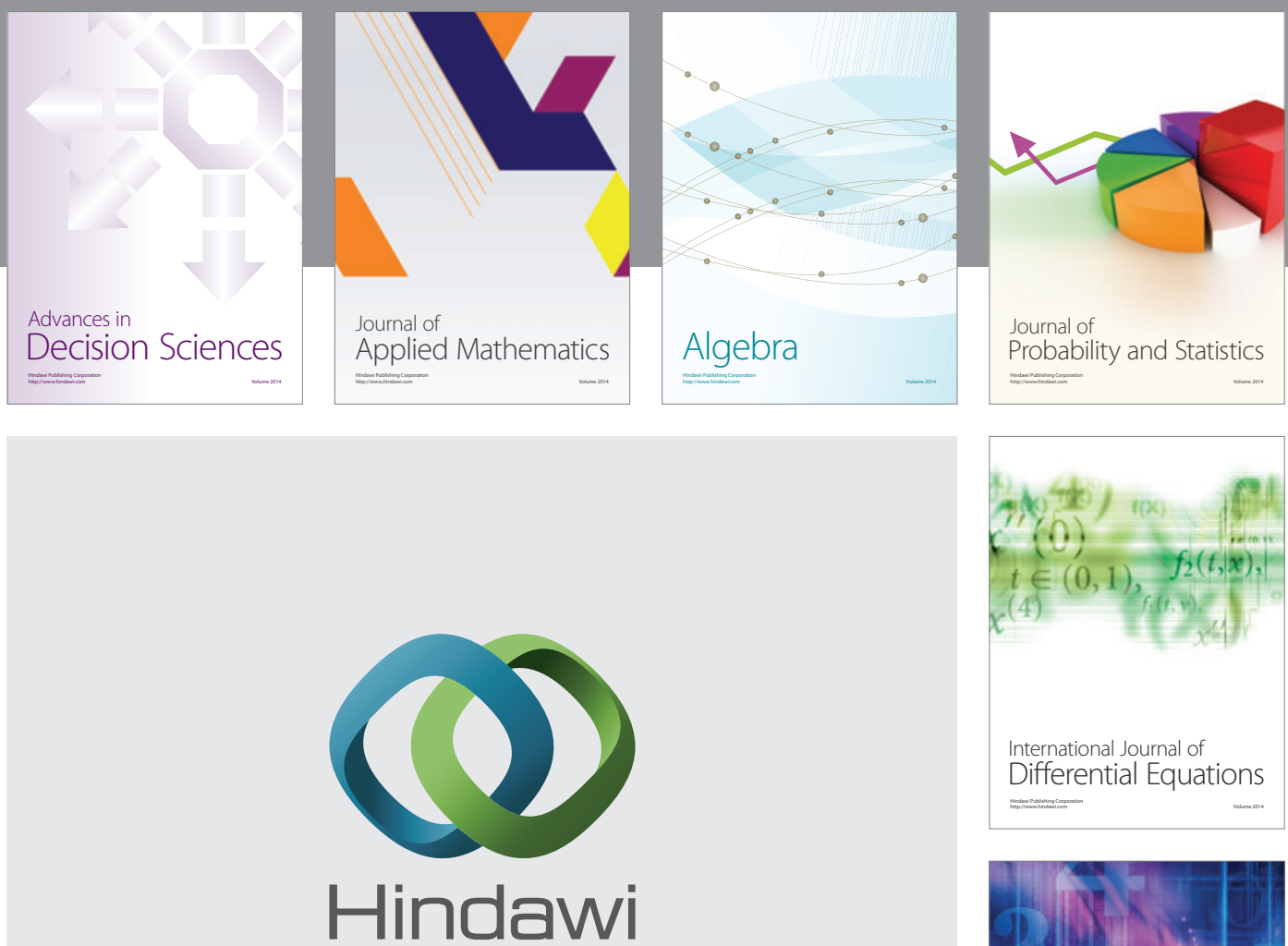

Submit your manuscripts at http://www.hindawi.com
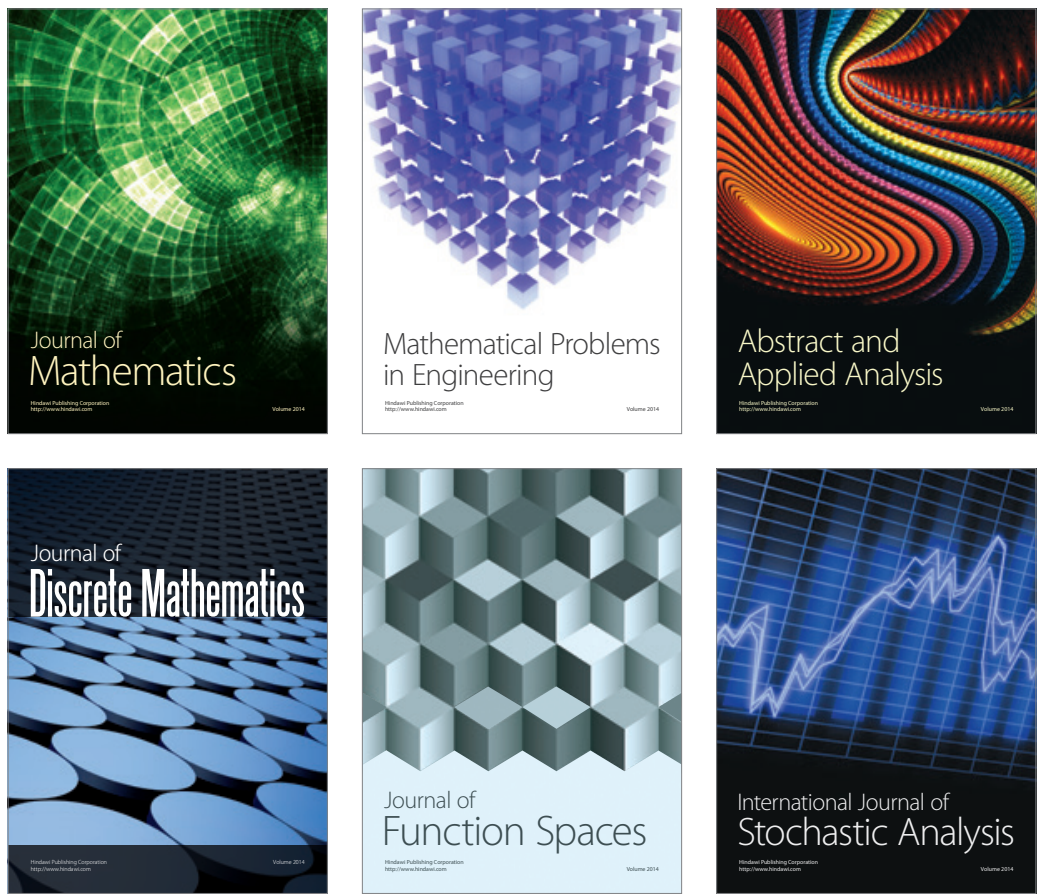

Journal of

Function Spaces

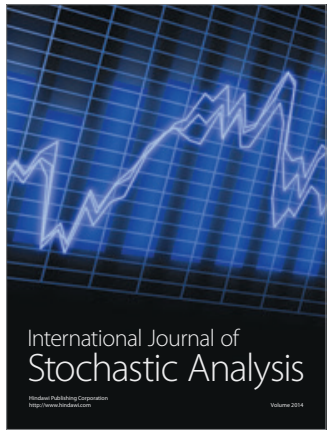

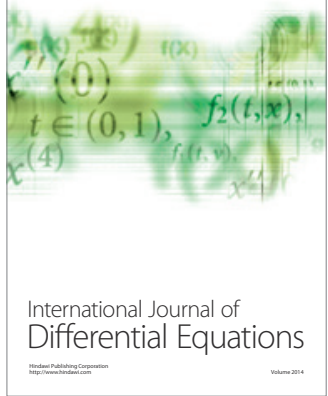
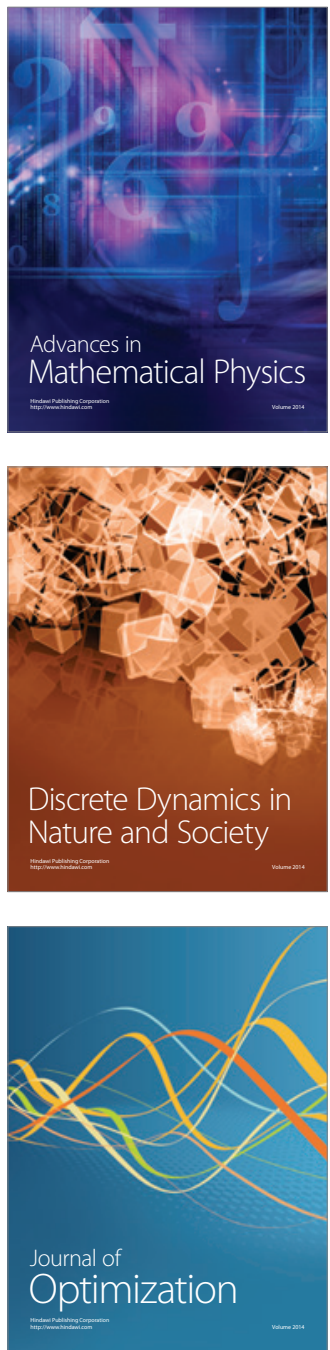